\title{
Semivariogram models for estimating fig fly population density throughout the year
}

\author{
Mauricio Paulo Batistella Pasini(1), Alessandro Dal'Col Lúcio(1) and Alberto Cargnelutti Filho(1) \\ (1)Universidade Federal de Santa Maria, Departamento de Fitotecnia, Avenida Roraima, № 1.000, Prédio 77, Cidade Universitária, Camobi, \\ CEP 97105-900 Santa Maria, RS, Brazil. E-mail: mauricio.pasini@gmail.com, adlucio@ufsm.br, alberto.cargnelutti.filho@gmail.com
}

\begin{abstract}
The objective of this work was to select semivariogram models to estimate the population density of fig fly (Zaprionus indianus; Diptera: Drosophilidae) throughout the year, using ordinary kriging. Nineteen monitoring sites were demarcated in an area of $8,200 \mathrm{~m}^{2}$, cropped with six fruit tree species: persimmon, citrus, fig, guava, apple, and peach. During a 24 month period, 106 weekly evaluations were done in these sites. The average number of adult fig flies captured weekly per trap, during each month, was subjected to the circular, spherical, pentaspherical, exponential, Gaussian, rational quadratic, hole effect, K-Bessel, J-Bessel, and stable semivariogram models, using ordinary kriging interpolation. The models with the best fit were selected by cross-validation. Each data set (months) has a particular spatial dependence structure, which makes it necessary to define specific models of semivariograms in order to enhance the adjustment to the experimental semivariogram. Therefore, it was not possible to determine a standard semivariogram model; instead, six theoretical models were selected: circular, Gaussian, hole effect, K-Bessel, J-Bessel, and stable.
\end{abstract}

Index terms: Zaprionus indianus, geostatistics, integrated pest management, monitoring, regionalized variables, spatial dependence.

\section{Modelos de semivariogramas para estimar a densidade populacional da mosca-do-figo ao longo do ano}

Resumo - O objetivo deste trabalho foi selecionar modelos de semivariogramas para estimar a densidade populacional da mosca-do-figo (Zaprionus indianus; Diptera: Drosophilidae) ao longo do ano, com uso da krigagem ordinária. Dezenove locais de monitoramento foram demarcados em área de $8.200 \mathrm{~m}^{2}$, cultivada com seis espécies de frutíferas: caquizeiro, citros, figueira, goiabeira, macieira e pessegueiro. Durante um período de 24 meses, foram realizadas 106 avaliações semanais nesses locais. O número médio de moscas-do-figo capturadas semanalmente por armadilha, por mês, foi submetido aos modelos de semivariogramas circular, esférico, pentaesférico, exponencial, gaussiano, quadrático racional, seno cardinal, K-Bessel, J-Bessel e estável, por meio de interpolação por krigagem ordinária. Os modelos com melhor ajuste foram selecionados a partir da validação cruzada. Cada conjunto de dados (meses) tem uma estrutura de dependência espacial específica, o que torna necessário definir modelos específicos de semivariogramas para melhorar o ajuste ao semivariograma experimental. Portanto, não foi possível definir um modelo padrão de semivariograma; ao invés disso, seis modelos teóricos foram selecionados: circular, gaussiano, seno cardinal, K-Bessel, J-Bessel e estável.

Termos para indexação: Zaprionus indianus, geoestatística, manejo integrado de pragas, monitoramento, variáveis regionalizadas, dependência espacial.

\section{Introduction}

The fig fly, Zaprionus indianus (Diptera: Drosophilidae), is a major pest in fig (Ficus carica L.) crops. It attacks fig fruit at the beginning of maturation, laying its eggs in the ostiole, which makes them unfit for consumption and processing. During the off-season, the fig fly searches for other hosts and is associated with decaying fruits. There is no information on the spatial distribution of the fig fly throughout the year, neither on the use of ordinary kriging to estimate its population density. According to Lasmar et al. (2012), the spatial distribution pattern of insects may vary over time, and its knowledge can help pest management by assisting in decision making, promoting local control of insect pest infestations, and reducing production costs with less use of insecticides, diminishing impacts on the environment. Therefore, 
the knowledge of pest behavior, feeding preferences, and habitats throughout the year is important for the establishment of an integrated pest management (Pasini et al., 2011).

Insect populations in orchards can be estimated by interpolation procedures, which generate continuous information surfaces through punctual sample units (Webster \& Oliver, 2007). Among the interpolation methods, ordinary kriging is one of the most used (Silva et al., 2010; Bottega et al., 2013). This method uses the spatial dependence among neighboring samples and estimates variable values in the semivariogram, at any position within the experimental area (Webster \& Oliver, 2007). Mello et al. (2005) highlight that the semivariogram has a pivotal importance for geostatistics, since it is able to describe the structure of spatial dependence, and it is crucial for determining the interpolator, having direct influence on the estimated values.

A valid semivariogram model has to be selected, and the model parameters have to be estimated before kriging (Gundogdu \& Guney, 2007). According to Webster \& Oliver (2007), the selection of a model that soundly represents the semivariances is very important for kriging. A wrong choice of a theoretical semivariogram model generates errors in the estimates, overestimating or underestimating values.

Gundogdu \& Guney (2007) tested the circular, spherical, tetraspherical, pentaspherical, exponential, Gaussian, rational quadratic, hole effect, K-Bessel, $\mathrm{J}$-Bessel, and stable models to determine underground water levels, and found that the rational quadratic one had the best fit. Farias et al. (2008) studied the spatial distribution of the fall armyworm (Spodoptera frugiperda) and observed that the spherical model had the best fit. Lasmar et al. (2012) determined the spatial distribution of ants in a eucalyptus (Eucalyptus spp.) plantation using the spherical, exponential, and Gaussian semivariogram models, and reported the best fit for the exponential model. Mora \& Beer (2013) used the spherical model to study the spatial variability of coffee (Coffea arabica L.) fine roots, under Erythrina shade. Noetzold et al. (2014) evaluated the spatial variability of Colletotrichum truncatum on soybean (Glycine $\max$ L.) field with the spherical, exponential, Gaussian, and K-Bessel models, and found the best adjustment for the spherical model. However, most studies with geostatistical models use few semivariograms and do not contemplate the possibility that data sets may show a different spatial structure with time.

The objective of this work was to select semivariogram models to estimate the population density of fig fly throughout the year, using ordinary kriging.

\section{Materials and Methods}

The study was carried out in the municipality of Santa Maria, in the state of Rio Grande do Sul, Brazil (29 43'26"S, 53 $\left.43^{\prime} 4^{\prime \prime} \mathrm{W}\right)$, in $8,200 \mathrm{~m}^{2}$, cropped with six fruit tree species: persimmon (Diospyros kaki L.), citrus (Citrus spp.), fig, guava (Psidium guajava L.), apple (Malus domestica Borkh.), and peach [Prunus persica (L.) Batsch]. The plants were eight-years-old, spaced at $2 \times 3 \mathrm{~m}$, and the plots were distributed in bands of length varying from 50 to $80 \mathrm{~m}$. According to Köppen's classification, local climate is Cfa, subtropical humid, without dry season and with hot summers (Heldwein et al., 2009).

Nineteen monitoring points were randomly distributed in the plots, using one trap for each $430 \mathrm{~m}^{2}$. The monitoring sites were demarcated with the aid of a global navigation satellite system (GNSS) receiver, which registered their geographical coordinates. At each monitoring point, a bottle fly trap was placed on a fruit tree $0.5 \mathrm{~m}$ above ground and protected from the sunlight from the east side of the canopy. The traps were composed of 0.6 L PET bottles, with two $0.8 \mathrm{~cm}$ holes to allow the entry of insects. The bottle traps contained attractive solution of fig juice and water $(50: 50 \%)$ with a total volume of $200 \mathrm{~mL}$. Each trap was used for a 28 day period, as in Pasini et al. (2011). The traps remained in the field from September $30^{\text {th }}$, 2009 , to October $4^{\text {th }}, 2011$, and the trapped insects were collected weekly and screened. A total of 106 evaluations were performed. Adult fig fly individuals were identified and quantified based on Yassin \& David (2010).

The number of adult fig flies captured weekly by the traps was organized accordingly to the 24 month period. The following descriptive statistics were estimated for each month: average, median, standard deviation, standard error, kurtosis, asymmetry, and coefficient of variation. Data with normal distribution or that showed negative asymmetry was not transformed (Yamamoto 
\& Landin, 2013). The hypothesis of normality of the data was tested by the Anderson-Darling test, at 5\% probability, and, if not satisfied, the data were subjected to the Box-Cox transformation (Box \& Cox, 1964). Then, the data were subjected to geostatistical analysis in order to verify the existence of spatial dependence; if existent, its degree was quantified by comparing the models to the isotropic experimental semivariogram, estimated by

$$
\gamma(\mathrm{h})=\frac{1}{2 \mathrm{~N}(\mathrm{~h})} \sum_{\mathrm{i}=1}^{\mathrm{N}(\mathrm{h})}\left[\mathrm{Z}\left(\mathrm{x}_{\mathrm{i}}\right)-\mathrm{Z}\left(\mathrm{x}_{\mathrm{i}}+\mathrm{h}\right)\right]^{2},
$$

in which (h) is the semivariance and $N(h)$ is the number of $\mathrm{Z}\left(\mathrm{x}_{\mathrm{i}}\right)$ and $\mathrm{Z}\left(\mathrm{x}_{\mathrm{i}}+\mathrm{h}\right)$ pairs measured, separated by an $\mathrm{h}$ vector.

From the experimental semivariograms, 11 theoretical models of semivariograms were adjusted (Johnson et al., 2001): circular,

$$
\gamma(h)=\frac{2 C_{0}+C_{1}}{\pi}\left[\frac{h}{a} \sqrt{1-\left(\frac{h}{a}\right)^{2}}+\arcsin \frac{h}{a}\right]
$$

for $0 \leq \mathrm{h} \leq \mathrm{a}$, and $\gamma(\mathrm{h} ; \theta)=\mathrm{C}_{0}+\mathrm{C}_{1}$ for $\mathrm{a}<\mathrm{h}$; spherical,

$$
\gamma(\mathrm{h})=\mathrm{C}_{0}+\mathrm{C}_{1}\left[\frac{3}{2} \frac{\mathrm{h}}{\mathrm{a}}-\frac{1}{2}\left(\frac{\mathrm{h}}{\mathrm{a}}\right)^{3}\right]
$$

for $0 \leq \mathrm{h} \leq \mathrm{a}$, and $\gamma(\mathrm{h} ; \theta)=\mathrm{C}_{0}+\mathrm{C}_{1}$ for $\mathrm{a}<\mathrm{h}$; pentaspherical,

$$
\gamma(\mathrm{h})=\mathrm{C}_{0}+\mathrm{C}_{1}\left[\frac{15}{8} \frac{\mathrm{h}}{\mathrm{a}}-\frac{5}{4}\left(\frac{\mathrm{h}}{\mathrm{a}}\right)^{3}+\frac{3}{8}\left(\frac{\mathrm{h}}{\mathrm{a}}\right)^{5}\right]
$$

for $0 \leq \mathrm{h} \leq \mathrm{a}$, and $\gamma(\mathrm{h} ; \theta)=\mathrm{C}_{0}+\mathrm{C}_{1}$ for $\mathrm{a}<\mathrm{h}$; exponential,

$$
\gamma(\mathrm{h})=\mathrm{C}_{0}+\mathrm{C}_{1}\left[1-\mathrm{e}^{-3\left(\frac{\mathrm{h}}{\mathrm{a}}\right)}\right]
$$

for all h; Gaussian,

$$
\gamma(\mathrm{h})=\mathrm{C}_{0}+\mathrm{C}_{1}\left[1-\mathrm{e}^{-3\left(\frac{\mathrm{h}}{\mathrm{a}}\right)^{2}}\right]
$$

for all h; rational quadratic,

$$
\gamma(h)=C_{0}+C_{1} \frac{19\left(\frac{h}{a}\right)^{2}}{1+19\left(\frac{h}{a}\right)^{2}}
$$

for all $\mathrm{h}$; hole effect, 0 for $\mathrm{h}=0$ and

$$
\gamma(h)=C_{0}+C_{1} \frac{1-\sin \left(\frac{2 \pi h}{a}\right)}{\sin \left(\frac{2 \pi h}{a}\right)}
$$

for $\mathrm{h} \neq 0$; K-Bessel,

$$
\gamma(\mathrm{h})=\mathrm{C}_{0}+\mathrm{C}_{1}\left[1-\frac{\left(\frac{\Omega_{\theta_{\mathrm{k}}} \mathrm{h}}{\mathrm{a}}\right)^{\theta_{\mathrm{k}}}}{2^{\theta_{\mathrm{k}}-1} \Gamma\left(\theta_{\mathrm{k}}\right)} \mathrm{K}_{\theta_{\mathrm{k}}}\left(\frac{\Omega_{\theta_{\mathrm{k}}} \mathrm{h}}{\mathrm{a}}\right)\right]
$$

for all h, in which $\Omega_{\theta \mathrm{k}}$ is a value found numerically so that $\gamma(\mathrm{a})-0.95\left(\mathrm{C}_{0}+\mathrm{C}_{1}\right)$ for any $\theta_{\mathrm{k}}, \Gamma\left(\theta_{\mathrm{k}}\right)$ is the gamma function,

$$
\Gamma(y)=\int_{0}^{\infty} x y^{-1} \exp (-x) d x,
$$

and $\mathrm{K}_{\theta \mathrm{k}}$ is the modified Bessel function of the second kind of order $\theta_{\mathrm{k}}$; J-Bessel,

$$
\gamma(\mathrm{h})=\mathrm{C}_{0}+\mathrm{C}_{1}\left[1-\frac{2^{\theta_{\mathrm{d}}} \Gamma\left(\theta_{\mathrm{d}}+1\right)}{\left(\frac{\Omega_{\theta_{\mathrm{d}}} \mathrm{h}}{\mathrm{a}}\right)^{\theta_{\mathrm{d}}}} \mathrm{J}_{\theta_{\mathrm{d}}}\left(\frac{\Omega_{\theta_{\mathrm{d}}} \mathrm{h}}{\mathrm{a}}\right)\right]
$$

for all $\mathrm{h}$, in which $\mathrm{C}_{0}+\mathrm{C}_{1} \geq 0, \mathrm{a} \geq 0, \theta \geq 0, \Omega_{\theta_{\mathrm{d}}}$ must satisfy $B=a, B>0, \gamma(B)=C_{0}+C_{1}, \gamma(B)=C_{0}+C_{1}$, $\gamma^{\prime}(B)<0$, and $\mathrm{J}_{\theta_{\mathrm{d}}}$ is the J-Bessel function; and stable,

$$
\gamma(\mathrm{h})=\mathrm{C}_{0}+\mathrm{C}_{1}\left[1-\mathrm{e}^{\left(-3\left(\frac{\mathrm{h}}{\mathrm{a}}\right)^{\theta} \mathrm{e}\right)}\right]
$$

for all $\mathrm{h}$, in which $\mathrm{C}_{0}+\mathrm{C}_{1}$ and $0 \leq \theta_{\mathrm{e}} \leq 2$.

Using the algorithm of weighted least squares, these models were adjusted to the experimental semivariogram, and the following model parameters were defined: nugget effect $\left(\mathrm{C}_{0}\right)$, sill $\left(\mathrm{C}_{0}+\mathrm{C}_{1}\right)$, and range (a).

In order to verify the existence of spatial dependence, the spatial dependence index (SDI) was applied, which is the ratio representing the percentage of data variability explained by spatial dependence. The SDI is estimated with the expression: $\mathrm{SDI}=[\mathrm{C} 1 /(\mathrm{C} 0+\mathrm{C} 1)] 100$, being classified as strong $(\mathrm{SDI}>75 \%)$, medium $(25<\mathrm{SDI} \leq 75 \%)$, and low $(\mathrm{SDI} \leq 25 \%)$.

After the confirmation of spatial dependence, inferences were performed by ordinary kriging, following Johnson et al. (2001), which allowed for the 
estimation of values at locations not measured. For ordinary kriging, non-biased estimates, with minimum deviation from the known values, are interpolated, considering the spatial variability structure of the attribute (Webster \& Oliver, 2007).

The semivariogram model was selected according to the Webster \& Oliver (2007) cross-validation technique. Goovaerts (1997) argues that cross-validation allows comparing the impact of interpolators among the real estimated values, in which the model with more accurate predictions is chosen. Faraco et al. (2008) considered the cross-validation criterion as the most adequate for choosing the best semivariogram adjustment. Linear regression was used as a first indicator of cross-validation, in which the estimated values (dependent variable) were crossed with the sampled values (independent variable). The best adjustments are obtained when the estimation of the intercept a approaches zero, and the linear $b$ and determination $\mathrm{R}^{2}$ coefficients approach 1 .

As a second indicator, the mean prediction error $(\overline{\mathrm{E}})$ was used, estimated by the expression:

$$
\overline{\mathrm{E}}=\frac{\sum_{\mathrm{i}=1}^{\mathrm{n}}\left[\hat{\mathrm{Z}}\left(\mathrm{s}_{\mathrm{i}}\right)-\mathrm{z}\left(\mathrm{s}_{\mathrm{i}}\right)\right]}{\mathrm{n}},
$$

in which $\mathrm{z}$ is the observed value and $\hat{z}$ is the estimated value. As a third indicator, the standard deviation of the prediction error (SD) was used, estimated by the expression:

$$
\mathrm{SD}=\sqrt{\frac{\sum_{\mathrm{i}=1}^{\mathrm{n}}\left\{\left[\hat{\mathrm{Z}}\left(\mathrm{s}_{\mathrm{i}}\right)-\mathrm{z}\left(\mathrm{s}_{\mathrm{i}}\right)\right]-\mathrm{E}\right\}^{2}}{\mathrm{n}-1} .}
$$

As a fourth indicator, the coefficient of variation (VC) was used, and as a fifth, the mean prediction absolute error $(\overline{\mathrm{A}} \overline{\mathrm{E}})$, estimated by the expression:

$$
\overline{\mathrm{AE}}=\frac{\sum_{\mathrm{i}=1}^{\mathrm{n}}\left|\left[\hat{\mathrm{Z}}\left(\mathrm{s}_{\mathrm{i}}\right)-\mathrm{z}\left(\mathrm{s}_{\mathrm{i}}\right)\right]\right|}{\mathrm{n}} \text {. }
$$

The root-mean-square prediction error (RMS) was used as a sixth indicator, estimated by the expression:

$$
\mathrm{RMS}=\sqrt{\frac{\sum_{\mathrm{i}=1}^{\mathrm{n}}\left[\hat{\mathrm{Z}}\left(\mathrm{s}_{\mathrm{i}}\right)-\mathrm{z}\left(\mathrm{s}_{\mathrm{i}}\right)\right]^{2}}{\mathrm{n}}} .
$$

Pesq. agropec. bras., Brasília, v.49, n.7, p.493-505, jul 2014 DOI: 10.1590/S0100-204X2014000700001
For these parameters, the closer to zero, the best the adjustment of the model. As a seventh indicator, the root-mean-square standardized prediction error (RMSS) was used, estimated by the expression:

$$
\mathrm{RMSS}=\sqrt{\frac{\sum_{\mathrm{i}=1}^{\mathrm{n}}\left[\left(\hat{\mathrm{Z}}\left(\mathrm{s}_{\mathrm{i}}\right)-\mathrm{z}\left(\mathrm{s}_{\mathrm{i}}\right)\right) / \sigma\left(\mathrm{s}_{\mathrm{i}}\right)\right]^{2}}{\mathrm{n}} .}
$$

For this parameter, the best adjustment is obtained when it approaches 1 .

The cross-validation grades for the indicators varied from 1 to 10 , according to the selected criterion of each indicator: for $b, R^{2}$, and RMSS, values closer to 1 received the grade 10 , whereas the most distant values received the grade 1; for the estimates $\overline{\mathrm{E}}, \mathrm{SD}, \mathrm{VC}$, $\bar{A} \bar{E}$, and RMS, values closer or equal to zero received the grade 10, and the most distant values received the grade 1 . The model with the highest sum of grades was chosen.

\section{Results and Discussion}

During the 24 month monitoring period, 47,193 adult fig flies were captured, with weekly average of 23.5 adults captured per trap. In the first 12 months, 23,104 adult fig flies were captured, with weekly average of 22.9 adults per trap. In the last 12 months, 24,089 adult flies were captured, with a daily average of 23.9 adults per trap. These weekly averages were lower than the ones reported by Raga et al. (2006), Pasini et al. (2011), Pasini \& Link (2011), but they represent a considerably longer period.

During the two experimental years, the highest capture levels were registered in March, with 79.2 and 84.4 fig fly adults per trap per week in the first and in the second year, respectively. April had the next higher capture levels, with 51.1 and 49 adults per trap per week, respectively. These values are high when compared to those found by Raga et al. (2006), Pasini et al. (2011), and Pasini \& Link (2011). Moreover, they were obtained during the maturation period of the figs and, therefore, represent a high damage threat for the fruit (Table 1). August and September were the months with lower capture rate, in both years, possibly because of the lack of food and low temperatures. During these months, the average weekly number of adult individuals captured per trap was inferior to seven. 
The collected data were rather variable, in both years, as attested by the high coefficient of variation $(\mathrm{CV} \geq 48 \%)$ (Table 1). This variability is possibly explained by the great diversity of fruit trees existing in the orchard. According to Pasini et al. (2011), fig fly has a great number of hosts and its development is close to the substrate, which influences the distribution of pest population in the orchards and favors the presence of outliers.

In all tested semivariograms, theoretical models show strong spatial dependence (Tables 2 and 3), which can highly contribute to data variability and, therefore, may be used in the ordinary kriging interpolator.

After the interpolation, semivariogram models with higher sum for cross-validation indicators were chosen (Tables 4 and 5). Different semivariogram models were obtained for the different months and years, which agrees with the hypothesis of Gundogdu \& Guney (2007) that each data set presents a different spatial structure and that it is necessary to define a semivariogram model with the best fit to each one of them.

In the first year, the circular model had a higher sum of cross-validation indicators in December, June, and July; the hole effect model, in October, January, March, and August; and the stable model, in November. In the second year, the Gaussian model had the best fit in October, November, December, February, July, and August; the hole effect model, in January, March, May, and September; the K-Bessel model, in April; and the J-Bessel model, in June. This result does not agree with those reported by other authors, who found that the spherical and exponential

Table 1. Descriptive statistics of the weekly average number of fig flies (Zaprionus indianus; Diptera: Drosophilidae) captured over different months, in the first and second years of monitoring.

\begin{tabular}{|c|c|c|c|c|c|c|c|c|c|c|c|c|}
\hline Statistics & Oct. & Nov. & Dec. & Jan. & Feb. & Mar. & Apr. & May & Jun. & Jul. & Aug. & Sep. \\
\hline & \multicolumn{12}{|c|}{ First year } \\
\hline Average & 6.9 & 12.3 & 16.7 & 20.1 & 25.5 & 79.2 & 51.1 & 29.2 & 19.9 & 6.0 & 3.1 & 3.6 \\
\hline Median & 5.5 & 10.3 & 14.0 & 15.8 & 23.8 & 36.6 & 46.5 & 27.8 & 14.2 & 4.5 & 3.0 & 2.8 \\
\hline Minimum & 1.8 & 5.8 & 5.2 & 8.5 & 7.0 & 8.4 & 8.3 & 2.0 & 2.2 & 1.0 & 0.8 & 0.3 \\
\hline Maximum & 18.3 & 28.3 & 39.2 & 45.0 & 53.8 & 306.8 & 153.8 & 54.5 & 59.8 & 14.5 & 7.0 & 13.5 \\
\hline Interval & 16.5 & 22.5 & 34.0 & 36.5 & 46.8 & 298.4 & 145.5 & 52.5 & 57.6 & 13.5 & 6.2 & 13.3 \\
\hline Standard deviation & 4.5 & 5.9 & 9.8 & 11.2 & 14.3 & 103.7 & 48.1 & 18.1 & 17.3 & 4.3 & 1.6 & 3.5 \\
\hline Standard error & 1.0 & 1.4 & 2.2 & 2.6 & 3.3 & 23.8 & 11.0 & 4.2 & 4.0 & 1.0 & 0.4 & 0.8 \\
\hline Kurtosis & 0.9 & 1.8 & 1.0 & 0.4 & -0.3 & 1.4 & 0.0 & -1.4 & 0.2 & -0.7 & 0.5 & 2.9 \\
\hline Asymmetry & 1.2 & 1.5 & 1.4 & 1.2 & 0.8 & 1.7 & 1.1 & 0.0 & 1.0 & 0.8 & 0.6 & 1.8 \\
\hline Coefficient of variation $(\%)$ & 65 & 48 & 58 & 55 & 56 & 131 & 94 & 62 & 87 & 72 & 50 & 97 \\
\hline Normality (p-value) & 0.04 & 0.00 & 0.00 & 0.01 & $0.08^{*}$ & 0.00 & 0.00 & $0.25^{*}$ & 0.04 & 0.04 & $0.73 *$ & 0.00 \\
\hline $\operatorname{Box}-\operatorname{Cox}(\lambda)$ & 0 & -0.58 & 0 & -0.63 & - & -0.33 & 0 & - & 0 & 0 & - & 0 \\
\hline \multirow[t]{2}{*}{ Normality (p-value) } & $0.76^{*}$ & $0.85^{*}$ & $0.51^{*}$ & $0.41^{*}$ & - & $0.11 *$ & $0.12 *$ & - & $0.42 *$ & $0.67 *$ & - & $0.86^{*}$ \\
\hline & \multicolumn{12}{|c|}{ Second year } \\
\hline Average & 8.5 & 15.0 & 20.4 & 19.8 & 26.9 & 84.4 & 49.5 & 26.0 & 15.7 & 7.2 & 5.0 & 4.9 \\
\hline Median & 5.0 & 11.6 & 14.0 & 15.0 & 25.0 & 49.8 & 66.5 & 33.4 & 13.8 & 5.0 & 3.2 & 3.0 \\
\hline Minimum & 0.8 & 1.2 & 1.3 & 8.5 & 8.5 & 8.8 & 1.5 & 1.6 & 2.5 & 0.8 & 0.8 & 0.6 \\
\hline Maximum & 29.5 & 36.8 & 46.8 & 45.8 & 58.8 & 317.4 & 117.8 & 43.6 & 40.3 & 23.3 & 11.6 & 14.2 \\
\hline Interval & 28.8 & 35.6 & 45.5 & 37.3 & 50.3 & 308.6 & 116.3 & 42.0 & 37.8 & 22.5 & 10.8 & 13.6 \\
\hline Standard deviation & 8.5 & 10.6 & 14.3 & 11.2 & 15.5 & 107.8 & 39.8 & 15.2 & 11.9 & 6.0 & 3.4 & 4.3 \\
\hline Standard error & 1.9 & 2.4 & 3.3 & 2.6 & 3.6 & 24.7 & 9.1 & 3.5 & 2.7 & 1.4 & 0.8 & 1.0 \\
\hline Kurtosis & 1.8 & -0.2 & -0.7 & 0.6 & 0.0 & 1.3 & -1.7 & -1.3 & 0.1 & 1.9 & -1.1 & 0.1 \\
\hline Asymmetry & 1.5 & 0.7 & 0.7 & 1.3 & 0.9 & 1.6 & 0.1 & -0.6 & 1.0 & 1.5 & 0.5 & 1.1 \\
\hline Coefficient of variation $(\%)$ & 100 & 70 & 70 & 56 & 57 & 127 & 80 & 58 & 76 & 82 & 67 & 87 \\
\hline Normality (p-value) & 0.00 & $0.15^{*}$ & 0.05 & 0.00 & 0.05 & 0.00 & 0.00 & 0.00 & 0.03 & 0.01 & 0.03 & 0.00 \\
\hline $\operatorname{Box}-\operatorname{Cox}(\lambda)$ & 0 & - & 0.43 & -0.78 & 0 & -0.28 & 0.33 & - & 0 & 0 & 0 & 0 \\
\hline Normality (p-value) & $0.87^{*}$ & - & $0.41 *$ & $0.45^{*}$ & $0.58 *$ & $0.09 *$ & $0.11 *$ & - & $0.51 *$ & $0.93 *$ & $0.20 *$ & $0.63 *$ \\
\hline
\end{tabular}

*Normal data, according to the Anderson-Darling test, at 5\% probability. Number of traps $=19$. 
Table 2. Nugget effect $\left(\mathrm{C}_{0}\right)$, sill $\left(\mathrm{C}_{1}\right)$, range (a), gamma function $(\Gamma)$, and spatial dependence index $(\mathrm{SDI})$ estimated in the first year of evaluation, for the following semivariogram models: C, circular; S, spherical; P, pentaspherical; E, exponential; G, Gaussian; R, rational quadratic; H, hole effect; K, K-Bessel; J, J-Bessel; and St, stable.

\begin{tabular}{|c|c|c|c|c|c|c|c|c|c|c|c|c|c|c|c|}
\hline Model & $\mathrm{C}_{0}$ & $\mathrm{C}_{1}$ & $\mathrm{a}$ & $\Gamma$ & $\mathrm{SDI}^{(1)}$ & $\mathrm{C}_{0}$ & $\mathrm{C}_{1}$ & $\mathrm{a}$ & $\Gamma$ & SDI & $\mathrm{C}_{0}$ & $\mathrm{C}_{1}$ & $\mathrm{a}$ & $\Gamma$ & SDI \\
\hline & \multicolumn{5}{|c|}{ October } & \multicolumn{5}{|c|}{ November } & \multicolumn{5}{|c|}{ December } \\
\hline $\mathrm{C}$ & 0 & 0.4334 & 42.2 & - & S & 0 & 0.0089 & 42.2 & - & S & 0 & 0.3437 & 78.1 & - & S \\
\hline S & 0 & 0.5043 & 32.3 & - & $\mathrm{S}$ & 0 & 0.0101 & 32.3 & - & $\mathrm{S}$ & 0 & 0.3681 & 97.7 & - & S \\
\hline $\mathrm{P}$ & 0.0034 & 0.4754 & 36.8 & - & $\mathrm{S}$ & 0 & 0.0093 & 32.9 & - & S & 0 & 0.3731 & 105.3 & - & S \\
\hline E & 0 & 0.4876 & 33.3 & - & $\mathrm{S}$ & 0.0002 & 0.0089 & 92.5 & - & $\mathrm{S}$ & 0 & 0.3659 & 105.3 & - & $\mathrm{S}$ \\
\hline G & 0 & 0.3639 & 42.2 & - & $\mathrm{S}$ & 0 & 0.0075 & 42.3 & - & $\mathrm{S}$ & 0 & 0.3147 & 47.8 & - & S \\
\hline $\mathrm{R}$ & 0.0005 & 0.4801 & 33.2 & - & $\mathrm{S}$ & 0.0007 & 0.0079 & 92.4 & - & $\mathrm{S}$ & 0 & 0.3621 & 119.1 & - & $\mathrm{S}$ \\
\hline $\mathrm{H}$ & 0 & 0.3875 & 42.2 & - & $\mathrm{S}$ & 0 & 0.0091 & 50.3 & - & $\mathrm{S}$ & 0 & 0.2567 & 65.5 & - & $\mathrm{S}$ \\
\hline K & 0 & 0.5432 & 32.1 & 2.03 & S & 0 & 0.0104 & 42.2 & 5.32 & $\mathrm{~S}$ & 0 & 0.3214 & 52.3 & 10 & $\mathrm{~S}$ \\
\hline $\mathrm{J}$ & 0 & 0.3993 & 40.7 & 0.36 & $\mathrm{~S}$ & 0 & 0.0089 & 56.9 & 0.07 & $\mathrm{~S}$ & 0 & 0.2902 & 65.8 & 4.25 & $\mathrm{~S}$ \\
\hline \multirow[t]{2}{*}{$\mathrm{St}$} & 0 & 0.5226 & 32.1 & - & $\mathrm{S}$ & 0 & 0.0075 & 42.2 & - & $\mathrm{S}$ & 0 & 0.3147 & 47.8 & - & $\mathrm{S}$ \\
\hline & \multicolumn{5}{|c|}{ January } & \multicolumn{5}{|c|}{ February } & \multicolumn{5}{|c|}{ March } \\
\hline $\mathrm{C}$ & 0 & 0.0072 & 80.4 & - & $\mathrm{S}$ & 0 & 289.06 & 91.1 & - & $\mathrm{S}$ & 0 & 0.2414 & 158.1 & - & $\mathrm{S}$ \\
\hline S & 0 & 0.0103 & 119.7 & - & $\mathrm{S}$ & 0 & 261.69 & 105.3 & - & $\mathrm{S}$ & 0 & 0.0723 & 59.1 & - & $\mathrm{S}$ \\
\hline $\mathrm{P}$ & 0 & 0.0082 & 105.3 & - & $\mathrm{S}$ & 0 & 269.07 & 105.3 & - & S & 0 & 0.1019 & 89.6 & - & $\mathrm{S}$ \\
\hline E & 0 & 0.0082 & 105.3 & - & $\mathrm{S}$ & 0 & 265.15 & 105.3 & - & $\mathrm{S}$ & 0 & 0.1451 & 105.3 & - & $\mathrm{S}$ \\
\hline G & 0 & 0.0074 & 48.2 & - & $\mathrm{S}$ & 0 & 258.53 & 56.1 & - & $\mathrm{S}$ & 0 & 0.0882 & 49.9 & - & $\mathrm{S}$ \\
\hline $\mathrm{R}$ & 0 & 0.0051 & 64.3 & - & S & 0.2344 & 234.44 & 78.9 & - & S & 0 & 0.1307 & 131.2 & - & $\mathrm{S}$ \\
\hline $\mathrm{H}$ & 0 & 0.0055 & 60.1 & - & $\mathrm{S}$ & 0 & 199.81 & 73.6 & - & $\mathrm{S}$ & 0 & 0.0097 & 47.8 & - & $\mathrm{S}$ \\
\hline K & 0 & 0.0101 & 90.1 & 10 & S & 0 & 257.11 & 90.2 & 0.96 & $\mathrm{~S}$ & 0 & 0.0657 & 46.1 & 10 & S \\
\hline $\mathrm{J}$ & 0 & 0.0078 & 105.3 & 3.39 & $\mathrm{~S}$ & 0 & 222.08 & 78.9 & 10 & S & 0 & 0.0761 & 47.4 & 3.6 & S \\
\hline \multirow[t]{2}{*}{$\mathrm{St}$} & 0 & 0.0101 & 119.1 & - & $\mathrm{S}$ & 0 & 270.01 & 90.5 & - & $\mathrm{S}$ & 0 & 0.0583 & 32.3 & - & $\mathrm{S}$ \\
\hline & \multicolumn{5}{|c|}{ April } & \multicolumn{5}{|c|}{ May } & \multicolumn{5}{|c|}{ June } \\
\hline $\mathrm{C}$ & 0 & 1.0361 & 60.3 & - & $\mathrm{S}$ & 0 & 250.1 & 40.2 & - & $\mathrm{S}$ & 0 & 1.3001 & 43.7 & - & $\mathrm{S}$ \\
\hline $\mathrm{S}$ & 0 & 0.9948 & 60.3 & - & $\mathrm{S}$ & 0 & 250.1 & 38.1 & - & $\mathrm{S}$ & 0 & 1.3021 & 49.3 & - & $\mathrm{S}$ \\
\hline $\mathrm{P}$ & 0 & 1.2821 & 105.3 & - & $\mathrm{S}$ & 0 & 243.7 & 42.2 & - & $\mathrm{S}$ & 0 & 1.1028 & 54.4 & - & $\mathrm{S}$ \\
\hline $\mathrm{E}$ & 0 & 1.2967 & 105.3 & - & $\mathrm{S}$ & 0 & 310.3 & 42.9 & - & $\mathrm{S}$ & 0 & 1.2534 & 59.7 & - & $\mathrm{S}$ \\
\hline G & 0 & 0.8029 & 47.3 & - & $\mathrm{S}$ & 0 & 210.1 & 42.2 & - & $\mathrm{S}$ & 0 & 1.9211 & 50.1 & - & S \\
\hline $\mathrm{R}$ & 0.0012 & 1.2652 & 105.3 & - & $\mathrm{S}$ & 0 & 324.2 & 60.8 & - & $\mathrm{S}$ & 0 & 1.4123 & 79.6 & - & $\mathrm{S}$ \\
\hline $\mathrm{H}$ & 0 & 0.8091 & 53.4 & - & $\mathrm{S}$ & 0 & 319.9 & 40.1 & - & $\mathrm{S}$ & 0 & 1.5141 & 65.6 & - & $\mathrm{S}$ \\
\hline K & 0 & 0.8686 & 51.9 & 10 & $\mathrm{~S}$ & 0 & 309.4 & 46.9 & 0.34 & S & 0 & 1.2678 & 60.1 & 0.57 & $\mathrm{~S}$ \\
\hline $\mathrm{J}$ & 0 & 0.8893 & 55.9 & 3.28 & $\mathrm{~S}$ & 0 & 280.2 & 50.1 & 10 & S & 0 & 0.9232 & 40.2 & 9.86 & $\mathrm{~S}$ \\
\hline \multirow[t]{2}{*}{$\mathrm{St}$} & 0 & 0.8029 & 56.5 & - & $\mathrm{S}$ & 0 & 309.5 & 46.6 & - & $\mathrm{S}$ & 0 & 1.2673 & 60.1 & - & $\mathrm{S}$ \\
\hline & \multicolumn{5}{|c|}{ July } & \multicolumn{5}{|c|}{ August } & \multicolumn{5}{|c|}{ September } \\
\hline $\mathrm{C}$ & 0 & 0.6529 & 42.2 & - & S & 0 & 3.1175 & 40.2 & - & S & 0 & 1.1203 & 54.9 & - & $\mathrm{S}$ \\
\hline S & 0 & 0.7022 & 42.2 & - & S & 0 & 3.1337 & 41.5 & - & S & 0 & 1.0731 & 58.8 & - & $\mathrm{S}$ \\
\hline $\mathrm{P}$ & 0 & 0.713 & 50.7 & - & S & 0 & 3.0217 & 42.2 & - & S & 0 & 1.2344 & 88.3 & - & $\mathrm{S}$ \\
\hline E & 0 & 0.7417 & 57.8 & - & $\mathrm{S}$ & 0 & 3.1036 & 46.1 & - & S & 0 & 1.3016 & 107.5 & - & $\mathrm{S}$ \\
\hline G & 0 & 0.6542 & 39.3 & - & S & 0 & 3.2561 & 42.2 & - & S & 0 & 1.0357 & 41.9 & - & $\mathrm{S}$ \\
\hline $\mathrm{R}$ & 0 & 0.7255 & 62.7 & - & S & 0 & 3.1081 & 52.5 & - & $\mathrm{S}$ & 0 & 1.2774 & 106.4 & - & $\mathrm{S}$ \\
\hline $\mathrm{H}$ & 0.0004 & 0.4244 & 59.2 & - & S & 0 & 2.7321 & 54.5 & - & S & 0 & 0.9116 & 69.8 & - & $\mathrm{S}$ \\
\hline K & 0 & 0.7318 & 42.2 & 1.96 & S & 0 & 3.2715 & 42.2 & 10 & S & 0 & 1.0561 & 45.1 & 9.93 & $\mathrm{~S}$ \\
\hline $\mathrm{J}$ & 0 & 0.6161 & 47.6 & 10 & $\mathrm{~S}$ & 0 & 2.6931 & 54.4 & 3.41 & $\mathrm{~S}$ & 0 & 1.0182 & 61.4 & 3.91 & S \\
\hline St & 0 & 0.7301 & 42.2 & - & S & 0 & 3.2847 & 34.5 & - & S & 0 & 1.0551 & 44.2 & - & S \\
\hline
\end{tabular}

${ }^{(1)}$ S, small, SDI $>75 \%$; M, medium, $25<\mathrm{SDI} \leq 75 \%$; L, large, $\mathrm{SDI} \leq 25 \%$. 
models predominate (Ellsbury et al., 1998; Farias et al., 2008; Dinardo-Miranda \& Fracasso, 2010; Lasmar et al., 2012). Gundogdu \& Guney (2007) evaluated a higher number of theoretical semivariograms and also observed a pattern of chosen models different than that reported in other works.

Table 3. Nugget effect $\left(\mathrm{C}_{0}\right)$, sill $\left(\mathrm{C}_{1}\right)$, range (a), gamma function $(\Gamma)$, and spatial dependence index (SDI) estimated in the second year of evaluation, for the following semivariogram models: $\mathrm{C}$, circular; $\mathrm{S}$, spherical; $\mathrm{P}$, pentaspherical; $\mathrm{E}$, exponential; G, Gaussian; R, rational quadratic; H, hole effect; K, K-Bessel; J, J-Bessel; and St, stable.

\begin{tabular}{|c|c|c|c|c|c|c|c|c|c|c|c|c|c|c|c|}
\hline Model & $\mathrm{C}_{0}$ & $\mathrm{C}_{1}$ & $\mathrm{a}$ & $\Gamma$ & $\mathrm{SDI}^{(1)}$ & $\mathrm{C}_{0}$ & $\mathrm{C}_{1}$ & $\mathrm{a}$ & $\Gamma$ & SDI & $\mathrm{C}_{0}$ & $\mathrm{C}_{1}$ & $\mathrm{a}$ & $\Gamma$ & SDI \\
\hline & \multicolumn{5}{|c|}{ October } & \multicolumn{5}{|c|}{ November } & \multicolumn{5}{|c|}{ December } \\
\hline $\mathrm{C}$ & 0 & 1.3901 & 32.1 & - & $\mathrm{S}$ & 0 & 154.74 & 42.2 & - & $\mathrm{S}$ & 0 & 10.46 & 42.2 & - & $\mathrm{S}$ \\
\hline $\mathrm{S}$ & 0 & 1.3751 & 33.2 & - & $\mathrm{S}$ & 0 & 149.71 & 45.2 & - & $\mathrm{S}$ & 0 & 13.7 & 48.5 & - & $\mathrm{S}$ \\
\hline $\mathrm{P}$ & 0 & 1.3803 & 33.2 & - & $\mathrm{S}$ & 0 & 116.69 & 40.8 & - & $\mathrm{S}$ & 0 & 10.67 & 49.5 & - & $\mathrm{S}$ \\
\hline $\mathrm{E}$ & 0 & 1.4401 & 40.2 & - & $\mathrm{S}$ & 0 & 117.41 & 40.3 & - & $\mathrm{S}$ & 0 & 9.02 & 40.2 & - & $\mathrm{S}$ \\
\hline G & 0 & 1.8020 & 42.2 & - & $\mathrm{S}$ & 0 & 145.78 & 42.2 & - & $\mathrm{S}$ & 0 & 10.31 & 42.2 & - & $\mathrm{S}$ \\
\hline $\mathrm{R}$ & 0 & 1.4185 & 40.2 & - & $\mathrm{S}$ & 0 & 168.72 & 83.7 & - & $\mathrm{S}$ & 0 & 8.86 & 32.2 & - & $\mathrm{S}$ \\
\hline $\mathrm{H}$ & 0 & 1.5323 & 44.3 & - & $\mathrm{S}$ & 0 & 109.34 & 52.9 & - & $\mathrm{S}$ & 0 & 9.34 & 48.2 & - & $\mathrm{S}$ \\
\hline $\mathrm{K}$ & 0 & 1.7536 & 42.2 & 2.52 & $\mathrm{~S}$ & 0 & 147.11 & 42.2 & 4.13 & $\mathrm{~S}$ & 0 & 10.64 & 42.2 & 1.44 & $\mathrm{~S}$ \\
\hline $\mathrm{J}$ & 0 & 1.7228 & 45.7 & 0.51 & $\mathrm{~S}$ & 0 & 117.51 & 49.7 & 0.04 & $\mathrm{~S}$ & 0 & 6.27 & 52.3 & 0.01 & $\mathrm{~S}$ \\
\hline \multirow[t]{2}{*}{$\underline{\mathrm{St}}$} & 0 & 1.8538 & 42.2 & - & $\mathrm{S}$ & 0 & 163.82 & 42.2 & - & $\mathrm{S}$ & 0 & 9.64 & 42.2 & - & $\mathrm{S}$ \\
\hline & \multicolumn{5}{|c|}{ January } & \multicolumn{5}{|c|}{ February } & \multicolumn{5}{|c|}{ March } \\
\hline $\mathrm{C}$ & 0 & 0.0038 & 98.3 & - & $\mathrm{S}$ & 0 & 0.4888 & 97.4 & - & $\mathrm{S}$ & 0 & 0.3369 & 105.3 & - & $\mathrm{S}$ \\
\hline $\mathrm{S}$ & 0 & 0.0041 & 105.3 & - & $\mathrm{S}$ & 0 & 0.4811 & 109.9 & - & $\mathrm{S}$ & 0 & 0.1145 & 68.5 & - & $\mathrm{S}$ \\
\hline $\mathrm{P}$ & 0 & 0.0041 & 149.1 & - & $\mathrm{S}$ & 0 & 0.4309 & 105.3 & - & $\mathrm{S}$ & 0 & 0.1103 & 51.1 & - & $\mathrm{S}$ \\
\hline $\mathrm{E}$ & 0 & 0.0032 & 105.3 & - & $\mathrm{S}$ & 0 & 0.4283 & 126.9 & - & $\mathrm{S}$ & 0 & 0.2011 & 142.1 & - & $\mathrm{S}$ \\
\hline $\mathrm{G}$ & 0 & 0.0031 & 105.3 & - & $\mathrm{S}$ & 0 & 0.0171 & 93.6 & - & $\mathrm{S}$ & 0 & 0.1872 & 85.1 & - & $\mathrm{S}$ \\
\hline $\mathrm{R}$ & 0 & 0.0021 & 51.9 & - & $\mathrm{S}$ & 0 & 0.2781 & 77.9 & - & $\mathrm{S}$ & 0 & 0.1968 & 105.3 & - & $\mathrm{S}$ \\
\hline $\mathrm{H}$ & 0 & 0.0029 & 136.1 & - & $\mathrm{S}$ & 0 & 0.4156 & 101.9 & - & $\mathrm{S}$ & 0 & 0.1111 & 49.9 & - & $\mathrm{S}$ \\
\hline $\mathrm{K}$ & 0 & 0.0039 & 100.3 & 10 & $\mathrm{~S}$ & 0 & 0.5987 & 136.1 & 1.81 & $\mathrm{~S}$ & 0 & 0.1246 & 50.1 & 10 & $\mathrm{~S}$ \\
\hline $\mathrm{J}$ & 0 & 0.0032 & 129.3 & 3.21 & $\mathrm{~S}$ & 0 & 0.3926 & 105.3 & 3.01 & $\mathrm{~S}$ & 0 & 0.1093 & 75.8 & 3.5 & $\mathrm{~S}$ \\
\hline \multirow[t]{2}{*}{$\underline{\mathrm{St}}$} & 0 & 0.0041 & 158.1 & - & $\mathrm{S}$ & 0 & 0.4932 & 105.3 & - & $\mathrm{S}$ & 0 & 0.1272 & 50.1 & - & $\mathrm{S}$ \\
\hline & \multicolumn{5}{|c|}{ April } & \multicolumn{5}{|c|}{ May } & \multicolumn{5}{|c|}{ June } \\
\hline $\mathrm{C}$ & 0 & 8.682 & 51.2 & - & $\mathrm{S}$ & 0 & 237.76 & 105.3 & - & $\mathrm{S}$ & 0 & 1.1981 & 87.1 & - & $\mathrm{S}$ \\
\hline S & 0 & 10.851 & 58.6 & - & $\mathrm{S}$ & 0 & 216.59 & 111.6 & - & $\mathrm{S}$ & 0 & 0.8855 & 67.5 & - & $\mathrm{S}$ \\
\hline $\mathrm{P}$ & 0 & 10.796 & 60.8 & - & $\mathrm{S}$ & 0 & 80.831 & 48.9 & - & $\mathrm{S}$ & 0 & 0.9912 & 84.3 & - & $\mathrm{S}$ \\
\hline $\mathrm{E}$ & 0 & 15.911 & 135.3 & - & $\mathrm{S}$ & 0 & 84.254 & 47.8 & - & $\mathrm{S}$ & 0 & 0.9624 & 105.3 & - & $\mathrm{S}$ \\
\hline $\mathrm{G}$ & 0 & 9.135 & 66.5 & - & $\mathrm{S}$ & 0 & 214.98 & 74.4 & - & $\mathrm{S}$ & 0 & 1.2011 & 78.1 & - & $\mathrm{S}$ \\
\hline $\mathrm{R}$ & 0 & 15.549 & 105.3 & - & $\mathrm{S}$ & 0 & 83.801 & 32.9 & - & $\mathrm{S}$ & 0.02 & 0.9161 & 105.3 & - & $\mathrm{S}$ \\
\hline $\mathrm{H}$ & 0 & 11.291 & 98.3 & - & $\mathrm{S}$ & 0 & 158.92 & 99.2 & - & $\mathrm{S}$ & 0 & 0.8441 & 81.3 & - & $\mathrm{S}$ \\
\hline K & 0 & 11.071 & 76.4 & 10 & $\mathrm{~S}$ & 0 & 223.43 & 81.1 & 10 & $\mathrm{~S}$ & 0 & 0.9258 & 74.6 & 1.01 & $\mathrm{~S}$ \\
\hline $\mathrm{J}$ & 0 & 10.064 & 95.67 & 3.31 & $\mathrm{~S}$ & 0 & 192.55 & 100.4 & 3.31 & $\mathrm{~S}$ & 0 & 0.9416 & 93.2 & 10 & $\mathrm{~S}$ \\
\hline \multirow[t]{2}{*}{$\mathrm{St}$} & 0 & 9.135 & 66.58 & - & $\mathrm{S}$ & 0 & 207.35 & 72.5 & - & $\mathrm{S}$ & 0 & 0.9294 & 74.1 & - & $\mathrm{S}$ \\
\hline & \multicolumn{5}{|c|}{ July } & \multicolumn{5}{|c|}{ August } & \multicolumn{5}{|c|}{ September } \\
\hline $\mathrm{C}$ & 0 & 1.0955 & 52.8 & - & $\mathrm{S}$ & 0 & 0.6881 & 42.3 & - & $\mathrm{S}$ & 0 & 0.8239 & 40.2 & - & $\mathrm{S}$ \\
\hline $\mathrm{S}$ & 0 & 0.9295 & 48.7 & - & $\mathrm{S}$ & 0 & 0.7098 & 50.1 & - & $\mathrm{S}$ & 0 & 0.8429 & 41.9 & - & $\mathrm{S}$ \\
\hline $\mathrm{P}$ & 0 & 0.9265 & 58.1 & - & $\mathrm{S}$ & 0 & 0.6968 & 55.4 & - & $\mathrm{S}$ & 0 & 0.0328 & 54.9 & - & $\mathrm{S}$ \\
\hline E & 0 & 0.9554 & 68.1 & - & $\mathrm{S}$ & 0 & 0.7407 & 66.1 & - & $\mathrm{S}$ & 0 & 0.9983 & 70.5 & - & $\mathrm{S}$ \\
\hline $\mathrm{G}$ & 0 & 1.0938 & 46.3 & - & $\mathrm{S}$ & 0 & 0.9739 & 48.7 & - & $\mathrm{S}$ & 0 & 1.2975 & 62.2 & - & $\mathrm{S}$ \\
\hline $\mathrm{R}$ & 0.038 & 0.8966 & 74.1 & - & $\mathrm{S}$ & 0 & 0.7333 & 73.3 & - & $\mathrm{S}$ & 0 & 0.9359 & 67.4 & - & $\mathrm{S}$ \\
\hline $\mathrm{H}$ & 0 & 0.9123 & 63.1 & - & $\mathrm{S}$ & 0 & 0.6876 & 55.4 & - & $\mathrm{S}$ & 0 & 0.8252 & 56.4 & - & $\mathrm{S}$ \\
\hline $\mathrm{K}$ & 0 & 0.9902 & 57.5 & 1.04 & $\mathrm{~S}$ & 0 & 0.7211 & 44.5 & 2.37 & $\mathrm{~S}$ & 0 & 0.9697 & 57.5 & 0.74 & $\mathrm{~S}$ \\
\hline $\mathrm{J}$ & 0 & 0.9084 & 59.7 & 10 & $\mathrm{~S}$ & 0 & 0.6726 & 53.4 & 2.57 & $\mathrm{~S}$ & 0 & 0.8481 & 56.1 & 1.02 & $\mathrm{~S}$ \\
\hline $\mathrm{St}$ & 0 & 0.9688 & 51.5 & - & $\mathrm{S}$ & 0 & 0.8213 & 51.1 & - & $\mathrm{S}$ & 0 & 0.8749 & 42.2 & - & $\mathrm{S}$ \\
\hline
\end{tabular}

${ }^{(1)} \mathrm{S}$, small, $\mathrm{SDI}>75 \%$; M, medium, $25<\mathrm{SDI} \leq 75 \%$; L, large, $\mathrm{SDI} \leq 25 \%$. 
Table 4. Cross-validation indicators and grades attributed (in brackets), in the first year of evaluation, obtained from ordinary kriging, for the following semivariogram models: $\mathrm{C}$, circular; S, spherical; P, pentaspherical; E, exponential; G, Gaussian; R, rational quadratic; $\mathrm{H}$, hole effect; K, K-Bessel; J, J-Bessel; and St, stable.

\begin{tabular}{|c|c|c|c|c|c|c|c|c|c|c|}
\hline Indicator $^{(1)}$ & $\mathrm{C}$ & $\mathrm{S}$ & $\mathrm{P}$ & $E$ & $\mathrm{G}$ & $\mathrm{R}$ & $\mathrm{H}$ & $\mathrm{K}$ & $\mathrm{J}$ & $\mathrm{St}$ \\
\hline & \multicolumn{10}{|c|}{ October } \\
\hline b & $0.5(8)$ & $0.3(3)$ & $0.2(1)$ & $0.2(1)$ & $0.6(10)$ & $0.2(1)$ & $0.3(3)$ & $0.3(3)$ & $0.3(3)$ & $0.2(1)$ \\
\hline $\mathrm{a}$ & $3.8(6)$ & $5.2(3)$ & $5.0(3)$ & $5.5(2)$ & $2.7(10)$ & $5.8(1)$ & $4.3(5)$ & $5.2(3)$ & $4.7(4)$ & $5.6(1)$ \\
\hline $\mathrm{R}^{2}$ & $0.4(10)$ & $0.3(5)$ & $0.3(5)$ & $0.2(1)$ & $0.4(10)$ & $0.2(1)$ & $0.4(10)$ & $0.3(5)$ & $0.4(10)$ & $0.3(5)$ \\
\hline$\overline{\mathrm{E}}$ & $0.0(10)$ & $-0.1(5)$ & $0.0(10)$ & $0.0(10)$ & $-0.2(1)$ & $0.0(10)$ & $0.1(5)$ & $-0.1(5)$ & $0.2(1)$ & $-0.1(5)$ \\
\hline SD & $3.6(7)$ & $3.7(6)$ & $3.8(5)$ & $3.9(4)$ & $4.2(1)$ & $4.1(3)$ & $3.4(10)$ & $3.7(6)$ & $3.5(8)$ & $3.9(4)$ \\
\hline $\mathrm{VC}$ & $340(3)$ & $68(5)$ & $384(2)$ & 391(1) & $21(10)$ & 257(4) & 23(9) & $57(7)$ & $19(10)$ & $67(6)$ \\
\hline$\overline{\mathrm{A}} \overline{\mathrm{E}}$ & $2.9(4)$ & $2.8(6)$ & $2.9(4)$ & $3.0(2)$ & $3.1(1)$ & $3.1(1)$ & $2.6(10)$ & $2.8(6)$ & $2.7(8)$ & $3.0(2)$ \\
\hline RMS & $3.5(8)$ & $3.6(6)$ & $3.7(4)$ & $3.8(3)$ & 4.1(1) & $3.9(2)$ & $3.4(10)$ & $3.6(6)$ & $3.4(10)$ & $3.8(3)$ \\
\hline RMSS & $0.9(10)$ & $0.6(3)$ & $0.6(3)$ & $0.6(3)$ & $1.4(6)$ & $0.6(3)$ & $0.7(1)$ & $0.6(3)$ & $0.6(3)$ & $0.6(3)$ \\
\hline \multirow[t]{2}{*}{$\sum$ notes } & 60 & 47 & 37 & 27 & 50 & 26 & 63 & 44 & 57 & 30 \\
\hline & \multicolumn{10}{|c|}{ November } \\
\hline b & $0.5(4)$ & $0.4(1)$ & $0.4(1)$ & $0.4(1)$ & $0.8(7)$ & $0.4(1)$ & $0.8(7)$ & $0.6(5)$ & $1.0(10)$ & $0.8(7)$ \\
\hline $\mathrm{a}$ & $5.7(6)$ & $6.7(5)$ & $7.4(1)$ & $7.2(2)$ & $2.8(8)$ & $7.0(4)$ & $2.2(9)$ & $4.3(7)$ & $0.6(10)$ & $2.8(8)$ \\
\hline $\mathrm{R}^{2}$ & $0.8(10)$ & $0.7(5)$ & $0.6(1)$ & $0.6(1)$ & $0.8(10)$ & $0.7(5)$ & $0.8(10)$ & $0.8(10)$ & $0.8(10)$ & $0.8(10)$ \\
\hline$\overline{\mathrm{E}}$ & $0.3(3)$ & $0.1(10)$ & $0.1(10)$ & $0.1(10)$ & $0.1(10)$ & $0.1(10)$ & $-0.2(6)$ & $0.1(10)$ & $-0.5(1)$ & $0.1(10)$ \\
\hline SD & $3.3(6)$ & $3.7(5)$ & $4.1(1)$ & $4.0(3)$ & $2.4(10)$ & $3.9(4)$ & $2.4(10)$ & $2.7(7)$ & $2.6(8)$ & $2.4(10)$ \\
\hline $\mathrm{VC}$ & $10(8)$ & $41(1)$ & $37(3)$ & $38(2)$ & 21(6) & $37(4)$ & $12(9)$ & $25(5)$ & $5(10)$ & $21(7)$ \\
\hline$\overline{\mathrm{A}} \overline{\mathrm{E}}$ & $2.6(6)$ & $2.9(4)$ & $3.2(1)$ & $3.1(2)$ & $2.0(10)$ & $3.0(3)$ & $2.0(10)$ & $2.3(8)$ & $2.0(10)$ & $2.0(10)$ \\
\hline RMS & $3.3(5)$ & $3.6(4)$ & $4.0(1)$ & $3.9(2)$ & $2.4(10)$ & $3.8(3)$ & $2.4(10)$ & $2.7(7)$ & $2.6(8)$ & $2.4(10)$ \\
\hline RMSS & $0.6(5)$ & $0.5(1)$ & $0.5(1)$ & $0.5(1)$ & $0.8(10)$ & $0.5(1)$ & $0.5(1)$ & $0.5(1)$ & $0.6(5)$ & $0.8(10)$ \\
\hline \multirow[t]{2}{*}{$\Sigma$ notes } & 44 & 36 & 20 & 24 & 81 & 35 & 62 & 50 & 72 & 84 \\
\hline & \multicolumn{10}{|c|}{ December } \\
\hline $\mathrm{b}$ & $0.6(3)$ & $0.6(3)$ & $0.6(3)$ & $0.5(1)$ & $0.8(10)$ & $0.7(6)$ & $0.7(6)$ & $0.7(6)$ & $0.8(10)$ & $0.8(10)$ \\
\hline $\mathrm{a}$ & $6.4(3)$ & $6.4(3)$ & $6.6(2)$ & $8.1(1)$ & $4.1(8)$ & $5.6(6)$ & $5.9(5)$ & $4.2(7)$ & $3.8(10)$ & $4.1(8)$ \\
\hline $\mathrm{R}^{2}$ & $0.9(10)$ & $0.8(5)$ & $0.8(5)$ & $0.7(1)$ & $0.7(1)$ & $0.7(1)$ & $0.8(5)$ & $0.7(1)$ & $0.7(1)$ & $0.7(1)$ \\
\hline$\overline{\mathrm{E}}$ & $0.2(1)$ & $0.2(1)$ & $0.2(1)$ & $0.1(5)$ & $0.0(10)$ & $0.2(1)$ & $0.1(5)$ & $0.0(10)$ & $-0.1(5)$ & $0.0(10)$ \\
\hline SD & $5.1(9)$ & $5.1(9)$ & $5.2(8)$ & $5.8(1)$ & $5.3(6)$ & $5.1(9)$ & $4.6(10)$ & $5.2(8)$ & $5.5(3)$ & $5.3(6)$ \\
\hline $\mathrm{VC}$ & $21(10)$ & $21(9)$ & $34(8)$ & $68(4)$ & $532(2)$ & $26(8)$ & $39(7)$ & $123(3)$ & $54(5)$ & $736(1)$ \\
\hline$\overline{\mathrm{A}} \overline{\mathrm{E}}$ & $3.3(8)$ & $3.4(6)$ & $3.4(6)$ & $3.9(1)$ & $3.4(6)$ & $3.2(10)$ & $3.2(10)$ & $3.3(8)$ & $3.5(4)$ & $3.4(6)$ \\
\hline RMS & $5.0(8)$ & $5.0(8)$ & $5.1(6)$ & $5.7(1)$ & $5.2(5)$ & $5.0(8)$ & $4.5(10)$ & $5.1(6)$ & $5.4(2)$ & $5.2(4)$ \\
\hline RMSS & $0.7(4)$ & $0.7(4)$ & $0.7(4)$ & $0.6(1)$ & $1.0(10)$ & $0.8(6)$ & $0.6(1)$ & $0.9(8)$ & $1.1(8)$ & $1.0(10)$ \\
\hline \multirow[t]{2}{*}{$\Sigma$ notes } & 60 & 48 & 42 & 16 & 58 & 55 & 59 & 57 & 48 & 54 \\
\hline & \multicolumn{10}{|c|}{ January } \\
\hline b & $0.6(8)$ & $0.6(8)$ & $0.5(6)$ & $0.4(4)$ & $0.5(6)$ & $0.3(2)$ & $0.6(8)$ & $0.7(10)$ & $0.1(1)$ & $0.5(6)$ \\
\hline $\mathrm{a}$ & $9.0(7)$ & $8.8(8)$ & $9.6(5)$ & $11.5(3)$ & $10.1(4)$ & $14.0(2)$ & $8.1(9)$ & $7.9(10)$ & 30.1(1) & $9.5(6)$ \\
\hline $\mathrm{R}^{2}$ & $0.6(10)$ & $0.6(10)$ & $0.5(8)$ & $0.5(8)$ & $0.2(2)$ & $0.3(6)$ & $0.6(10)$ & $0.1(2)$ & $0.0(1)$ & $0.5(8)$ \\
\hline$\overline{\mathrm{E}}$ & $0.3(8)$ & $-0.1(10)$ & $-0.1(10)$ & $-0.4(6)$ & $-1.6(3)$ & $0.3(8)$ & $0.5(4)$ & $-8.2(2)$ & $-29.3(1)$ & $-0.3(8)$ \\
\hline SD & $7.3(9)$ & $7.5(8)$ & $7.7(7)$ & $8.1(5)$ & $11.5(3)$ & $9.1(4)$ & $7.0(10)$ & $26.5(2)$ & 67.1(1) & $7.9(6)$ \\
\hline $\mathrm{VC}$ & $28(4)$ & $127(1)$ & $52(2)$ & $18(6)$ & $7(8)$ & $26(5)$ & $15(7)$ & $3(9)$ & $2(10)$ & $29(3)$ \\
\hline$\overline{\mathrm{A}} \overline{\mathrm{E}}$ & $5.0(9)$ & $5.1(8)$ & $5.3(7)$ & $5.9(5)$ & $7.8(3)$ & $6.6(4)$ & $4.9(10)$ & $19.0(2)$ & $45.3(1)$ & $5.4(6)$ \\
\hline RMS & $7.1(9)$ & $7.3(8)$ & $7.5(7)$ & $7.9(5)$ & $11.3(3)$ & $8.9(4)$ & $6.9(10)$ & $27.1(2)$ & $71.6(1)$ & $7.7(6)$ \\
\hline RMSS & $1.3(4)$ & $1.2(6)$ & $1.1(8)$ & $0.8(6)$ & $2.0(1)$ & $1.0(10)$ & $0.9(8)$ & $2.0(1)$ & $2.0(1)$ & $1.2(6)$ \\
\hline \multirow[t]{2}{*}{$\overline{\Sigma \text { notes }}$} & 68 & 67 & 60 & 48 & 33 & 45 & 76 & 40 & 18 & 55 \\
\hline & \multicolumn{10}{|c|}{ February } \\
\hline b & $0.7(10)$ & $0.6(5)$ & $0.6(5)$ & $0.6(5)$ & $0.7(10)$ & $0.5(1)$ & $0.7(10)$ & $0.7(10)$ & $0.7(10)$ & $0.6(5)$ \\
\hline $\mathrm{a}$ & $8.2(8)$ & $8.4(6)$ & $8.9(4)$ & $11.0(2)$ & $8.5(5)$ & $12.4(1)$ & $6.4(10)$ & $8.3(7)$ & $7.5(9)$ & $8.9(4)$ \\
\hline $\mathrm{R}^{2}$ & $0.7(10)$ & $0.7(10)$ & $0.7(10)$ & $0.6(1)$ & $0.7(10)$ & $0.6(1)$ & $0.7(10)$ & $0.7(10)$ & $0.7(10)$ & $0.6(1)$ \\
\hline$\overline{\mathrm{E}}$ & $0.6(4)$ & $0.6(4)$ & $0.6(4)$ & $0.3(6)$ & $-0.1(10)$ & $0.3(6)$ & $-0.4(4)$ & $-0.2(8)$ & $-0.4(6)$ & $0.7(1)$ \\
\hline SD & $8.3(5)$ & $8.3(5)$ & $8.5(4)$ & $8.7(3)$ & $7.8(10)$ & $9.4(1)$ & $8.1(7)$ & $7.9(9)$ & $8.1(7)$ & $8.8(2)$ \\
\hline $\mathrm{VC}$ & $13(10)$ & $14(8)$ & $15(7)$ & $28(4)$ & $77(1)$ & $33(3)$ & $22(5)$ & $36(2)$ & $22(6)$ & $13(10)$ \\
\hline$\overline{\mathrm{A}} \overline{\mathrm{E}}$ & $6.0(6)$ & $6.0(6)$ & $6.1(4)$ & $6.1(4)$ & $5.6(10)$ & $6.8(1)$ & $6.0(6)$ & $5.8(8)$ & $6.0(6)$ & $6.4(2)$ \\
\hline RMS & $8.1(5)$ & $8.1(5)$ & $8.3(4)$ & $8.5(3)$ & $7.6(10)$ & $9.1(1)$ & $7.8(7)$ & $7.7(8)$ & $7.9(6)$ & $8.6(2)$ \\
\hline RMSS & $0.9(10)$ & $0.9(10)$ & $0.9(10)$ & $0.8(1)$ & $0.8(1)$ & $0.8(1)$ & $0.9(10)$ & $0.8(1)$ & $0.9(10)$ & $0.9(10)$ \\
\hline \multirow[t]{2}{*}{$\sum$ notes } & 68 & 59 & 53 & 29 & 67 & 16 & 69 & 63 & 70 & 37 \\
\hline & & & & & & & & & & \\
\hline $\mathrm{b}$ & $0.6(1)$ & $0.6(1)$ & $0.6(1)$ & $0.6(1)$ & $0.6(1)$ & $0.8(10)$ & $0.7(5)$ & $0.8(10)$ & $0.6(1)$ & $0.6(1)$ \\
\hline $\mathrm{a}$ & $29.7(5)$ & $35.6(3)$ & $29.2(6)$ & $31.2(4)$ & $25.2(7)$ & $15.9(9)$ & $24.3(8)$ & $13.1(10)$ & $25.2(7)$ & $36.2(1)$ \\
\hline $\mathrm{R}^{2}$ & $0.5(10)$ & $0.5(10)$ & $0.5(10)$ & $0.5(10)$ & $0.5(10)$ & $0.5(10)$ & $0.5(10)$ & $0.5(10)$ & $0.4(1)$ & $0.5(10)$ \\
\hline$\overline{\mathrm{E}}$ & $-0.2(10)$ & $2.5(6)$ & $-1.9(7)$ & $-16.7(1)$ & $-8.9(3)$ & $-0.6(9)$ & $-3.1(5)$ & $1.0(8)$ & $-11.3(2)$ & $5.6(4)$ \\
\hline $\mathrm{SD}$ & $70.8(10)$ & $70.9(9)$ & $71.9(7)$ & $71.4(8)$ & $76.4(4)$ & $86.4(3)$ & $72.7(6)$ & $91.8(2)$ & 94.1(1) & $73.3(5)$ \\
\hline $\mathrm{VC}$ & $354(1)$ & $28(5)$ & $38(4)$ & $4(10)$ & $8(8)$ & $133(2)$ & $23(6)$ & $94(3)$ & $8(9)$ & $13(7)$ \\
\hline$\overline{\mathrm{A}} \overline{\mathrm{E}}$ & $40.6(6)$ & $38.9(10)$ & $42.1(5)$ & $49.7(2)$ & $39.7(8)$ & $45.8(4)$ & $40.5(7)$ & $46.7(3)$ & $51.5(1)$ & $39.6(9)$ \\
\hline RMS & $68.9(10)$ & $69.0(9)$ & $70.1(7)$ & $71.5(6)$ & $74.9(4)$ & 84.1(3) & $70.9(8)$ & $89.3(2)$ & $92.3(1)$ & $71.6(5)$ \\
\hline RMSS & $0.5(4)$ & $0.6(6)$ & $0.5(4)$ & $0.3(2)$ & $1.1(10)$ & $0.8(6)$ & $0.9(10)$ & $1.1(10)$ & $2.0(1)$ & $0.6(6)$ \\
\hline$\sum$ notes & 57 & 60 & 51 & 44 & 55 & 47 & 65 & 58 & 24 & 48 \\
\hline
\end{tabular}

Continues... 
Table 4. Continuation...

\begin{tabular}{|c|c|c|c|c|c|c|c|c|c|c|}
\hline \multirow[b]{2}{*}{ b } & \multicolumn{10}{|c|}{ April } \\
\hline & $0.7(5)$ & $0.7(5)$ & $0.7(5)$ & $0.7(5)$ & $0.8(7)$ & $0.2(1)$ & $0.8(7)$ & $0.8(7)$ & $0.8(7)$ & $0.9(10)$ \\
\hline $\mathrm{a}$ & $13.8(5)$ & $15.6(3)$ & $15.0(4)$ & $19.7(2)$ & $8.3(9)$ & $31.3(1)$ & $12.7(7)$ & $8.5(8)$ & $12.9(6)$ & $4.5(10)$ \\
\hline $\mathrm{R}^{2}$ & $0.8(7)$ & $0.8(7)$ & $0.8(7)$ & $0.8(7)$ & $0.9(10)$ & $0.1(1)$ & $0.7(5)$ & $0.9(10)$ & $0.9(10)$ & $0.8(7)$ \\
\hline$\overline{\mathrm{E}}$ & $0.3(7)$ & $-0.7(6)$ & $0.1(10)$ & $-2.8(2)$ & $1.3(5)$ & $2.3(3)$ & $-4.9(1)$ & $1.7(4)$ & $-0.3(7)$ & $-0.2(8)$ \\
\hline SD & 22.1(4) & $22.1(4)$ & $21.8(5)$ & $24.0(2)$ & $16.8(9)$ & 17.1(8) & $29.7(1)$ & 16.1(10) & $18.3(7)$ & $21.3(6)$ \\
\hline $\mathrm{VC}$ & $65(3)$ & $30(5)$ & $292(1)$ & $8(8)$ & $12(6)$ & $7(9)$ & $6(10)$ & $9(7)$ & $53(4)$ & $122(2)$ \\
\hline$\overline{\mathrm{E}}$ & $15.4(5)$ & $15.9(4)$ & $15.4(5)$ & $17.7(3)$ & $12.0(9)$ & $14.3(7)$ & $19.6(1)$ & $11.2(10)$ & $12.3(8)$ & $15.2(6)$ \\
\hline RMS & $21.6(3)$ & $21.5(4)$ & $21.3(5)$ & $23.6(2)$ & $16.4(9)$ & $16.8(8)$ & $29.3(1)$ & $15.8(10)$ & $17.9(7)$ & $20.7(6)$ \\
\hline RMSS & $0.5(4)$ & $0.4(1)$ & $0.5(4)$ & $0.4(1)$ & $0.7(7)$ & $1.0(10)$ & $0.8(8)$ & $0.6(6)$ & $0.5(4)$ & $1.4(6)$ \\
\hline \multirow[t]{2}{*}{$\sum$ notes } & 43 & 39 & 46 & 32 & 71 & 48 & 41 & 72 & 60 & 61 \\
\hline & \multicolumn{10}{|c|}{ May } \\
\hline b & $0.2(10)$ & $0.2(10)$ & $0.2(10)$ & $0.2(10)$ & $0.2(10)$ & $0.2(10)$ & $0.2(10)$ & $0.2(10)$ & $0.2(10)$ & $0.2(10)$ \\
\hline $\mathrm{a}$ & $19.5(9)$ & $20.6(7)$ & $21.3(5)$ & $22.5(3)$ & $21.3(5)$ & $21.3(5)$ & $20.4(8)$ & $22.9(1)$ & $19.4(10)$ & $22.8(2)$ \\
\hline $\mathrm{R}^{2}$ & $0.1(1)$ & $0.1(1)$ & $0.1(1)$ & $0.1(1)$ & $0.1(1)$ & $0.1(1)$ & $0.1(1)$ & $0.1(1)$ & $0.2(10)$ & $0.1(1)$ \\
\hline$\overline{\mathrm{E}}$ & $3.5(1)$ & $2.9(3)$ & $2.4(6)$ & $1.7(8)$ & $2.6(5)$ & $2.3(7)$ & $2.7(4)$ & $1.5(10)$ & $3.4(2)$ & $1.5(10)$ \\
\hline SD & 17.1(8) & 17.1(8) & $17.2(6)$ & $17.4(4)$ & $17.4(4)$ & 17.1(8) & $17.2(6)$ & $17.5(2)$ & $16.9(10)$ & $17.5(1)$ \\
\hline $\mathrm{VC}$ & $4(10)$ & $6(8)$ & $7(5)$ & $10(3)$ & $6(6)$ & $7(4)$ & $6(7)$ & $11(1)$ & $5(9)$ & $11(2)$ \\
\hline$\overline{\mathrm{A}} \overline{\mathrm{E}}$ & $14.1(7)$ & $14.4(3)$ & $14.5(2)$ & $14.7(1)$ & $14.7(1)$ & $14.3(5)$ & $14.7(1)$ & $14.7(1)$ & $13.9(10)$ & $14.7(1)$ \\
\hline RMS & $17.0(3)$ & $16.9(7)$ & $16.9(7)$ & 17.1(1) & 17.1(1) & $16.8(10)$ & $17.0(3)$ & 17.1(1) & $16.8(10)$ & 17.1(1) \\
\hline RMSS & $1.3(1)$ & $1.1(5)$ & $1.1(5)$ & $1.0(10)$ & $1.1(5)$ & $1.0(10)$ & $1.0(10)$ & $1.0(10)$ & $1.1(5)$ & $1.0(10)$ \\
\hline$\overline{\Sigma \text { notes }}$ & 50 & 52 & 47 & 43 & 38 & 60 & 50 & 37 & 76 & 38 \\
\hline \multirow{2}{*}{ Indicador $^{(1)}$} & C & $\mathrm{S}$ & $\mathrm{P}$ & $\mathrm{E}$ & $\mathrm{G}$ & $\mathrm{R}$ & $\mathrm{H}$ & $\mathrm{K}$ & $\mathrm{J}$ & St \\
\hline & \multicolumn{10}{|c|}{ June } \\
\hline b & $0.3(7)$ & $0.2(3)$ & $0.2(3)$ & $0.1(1)$ & $0.6(10)$ & $0.2(3)$ & $0.2(3)$ & $0.1(1)$ & $0.1(1)$ & $0.1(1)$ \\
\hline $\mathrm{a}$ & $10.8(9)$ & $11.5(8)$ & $14.0(6)$ & $16.3(2)$ & $6.1(10)$ & $13.6(7)$ & 16.1(3) & $15.7(5)$ & $18.6(1)$ & $15.7(5)$ \\
\hline $\mathrm{R}^{2}$ & $0.2(5)$ & $0.2(5)$ & $0.1(1)$ & $0.1(1)$ & $0.3(10)$ & $0.2(5)$ & $0.1(1)$ & $0.1(1)$ & $0.1(1)$ & $0.1(1)$ \\
\hline$\overline{\mathrm{E}}$ & $2.1(3)$ & $2.0(4)$ & $2.5(1)$ & $1.1(9)$ & $1.8(6)$ & $2.1(3)$ & $-0.5(10)$ & $1.4(8)$ & $1.9(5)$ & $1.4(8)$ \\
\hline SD & $15.9(9)$ & $16.0(8)$ & $16.1(7)$ & $16.7(3)$ & $16.9(1)$ & $15.8(10)$ & $16.6(4)$ & $16.5(5)$ & $16.8(2)$ & $16.5(5)$ \\
\hline $\mathrm{VC}$ & $7(9)$ & $8(7)$ & $6(10)$ & $14(2)$ & $9(5)$ & $7(8)$ & $31(1)$ & $12(3)$ & $8(6)$ & $12(4)$ \\
\hline $\bar{A} \overline{\mathrm{E}}$ & $11.6(8)$ & $11.9(7)$ & $12.0(6)$ & $12.8(2)$ & $10.8(10)$ & $11.6(8)$ & $12.8(2)$ & $12.6(4)$ & 13.1(1) & $12.6(4)$ \\
\hline RMS & $15.6(9)$ & $15.7(8)$ & $15.9(7)$ & $16.3(3)$ & $16.5(1)$ & $15.5(10)$ & $16.2(4)$ & $16.1(6)$ & $16.4(2)$ & $16.2(4)$ \\
\hline RMSS & $0.9(10)$ & $0.8(7)$ & $0.9(10)$ & $0.7(4)$ & $0.9(10)$ & $0.7(4)$ & $0.6(1)$ & $0.7(4)$ & $0.7(4)$ & $0.7(4)$ \\
\hline \multirow[t]{2}{*}{$\Sigma$ notes } & 69 & 57 & 51 & 27 & 64 & 58 & 29 & 37 & 23 & 36 \\
\hline & \multicolumn{10}{|c|}{ July } \\
\hline b & $0.2(10)$ & $0.2(10)$ & $0.2(10)$ & $0.1(1)$ & $0.2(10)$ & $0.1(1)$ & $0.2(10)$ & $0.2(10)$ & $0.1(1)$ & $0.2(10)$ \\
\hline a & $4.5(10)$ & $4.6(7)$ & $4.7(5)$ & $5.0(1)$ & $4.5(10)$ & $4.9(3)$ & $4.6(7)$ & $4.7(5)$ & $4.9(3)$ & $4.7(5)$ \\
\hline $\mathrm{R}^{2}$ & $0.2(10)$ & $0.1(1)$ & $0.1(1)$ & $0.1(1)$ & $0.2(10)$ & $0.1(1)$ & $0.1(1)$ & $0.1(1)$ & $0.1(1)$ & $0.1(1)$ \\
\hline$\overline{\mathrm{E}}$ & $-0.3(10)$ & $0.3(10)$ & $0.3(10)$ & $0.3(10)$ & $-0.3(10)$ & $0.3(10)$ & $0.3(10)$ & $0.3(10)$ & $0.4(1)$ & $0.3(10)$ \\
\hline SD & $4.0(10)$ & $4.1(7)$ & $4.1(7)$ & $4.2(3)$ & $4.0(10)$ & $4.2(3)$ & $4.1(7)$ & $4.1(7)$ & $4.3(1)$ & $4.1(7)$ \\
\hline $\mathrm{VC}$ & $15(2)$ & $12(9)$ & $14(4)$ & $16(1)$ & $13(5)$ & $13(6)$ & 14(3) & $12(8)$ & $10(10)$ & $12(7)$ \\
\hline$\overline{\mathrm{A}} \overline{\mathrm{E}}$ & $3.1(10)$ & $3.3(5)$ & $3.3(5)$ & $3.4(3)$ & $3.2(7)$ & $3.4(3)$ & $3.2(7)$ & $3.3(5)$ & $3.5(1)$ & $3.3(5)$ \\
\hline RMS & $3.9(10)$ & $4.0(7)$ & $4.0(7)$ & 4.1(3) & $3.9(10)$ & 4.1(3) & $4.0(7)$ & $4.0(7)$ & $4.2(1)$ & $4.0(7)$ \\
\hline RMSS & $1.0(10)$ & $0.9(5)$ & $0.9(5)$ & $0.8(1)$ & $0.9(5)$ & $0.8(1)$ & $0.9(5)$ & $1.0(10)$ & $1.0(10)$ & $0.9(5)$ \\
\hline \multirow[t]{2}{*}{$\overline{\Sigma \text { notes }}$} & 82 & 61 & 54 & 24 & 77 & 31 & 57 & 64 & 29 & 57 \\
\hline & \multicolumn{10}{|c|}{ August } \\
\hline b & $0.3(3)$ & $0.3(3)$ & $0.2(1)$ & $0.2(1)$ & $0.5(10)$ & $0.2(1)$ & $0.5(10)$ & $0.4(7)$ & $0.5(10)$ & $0.4(7)$ \\
\hline $\mathrm{a}$ & $2.1(4)$ & 2.2(3) & $2.4(2)$ & $2.6(1)$ & $1.6(8)$ & $2.6(1)$ & $1.4(10)$ & $1.7(6)$ & $1.4(10)$ & $1.9(5)$ \\
\hline $\mathrm{R}^{2}$ & $0.5(7)$ & $0.5(7)$ & $0.4(3)$ & $0.3(1)$ & $0.6(10)$ & $0.3(1)$ & $0.6(10)$ & $0.6(10)$ & $0.5(7)$ & $0.5(7)$ \\
\hline$\overline{\mathrm{E}}$ & $0.0(10)$ & $0.0(10)$ & $0.0(10)$ & $0.0(10)$ & $0.1(1)$ & $0.0(10)$ & $0.1(1)$ & $0.1(1)$ & $0.1(1)$ & $0.1(1)$ \\
\hline SD & $1.2(6)$ & $1.2(6)$ & $1.3(4)$ & $1.4(1)$ & $1.0(10)$ & $1.4(1)$ & $1.1(8)$ & $1.1(8)$ & 1.1(8) & $1.1(8)$ \\
\hline $\mathrm{VC}$ & $62(5)$ & $93(3)$ & $401(1)$ & $92(4)$ & $11(8)$ & $222(2)$ & $7(9)$ & $15(7)$ & $7(10)$ & $20(6)$ \\
\hline $\bar{A} \bar{E}$ & $0.9(7)$ & $1.0(3)$ & $1.0(3)$ & 1.1(1) & $0.8(10)$ & 1.1(1) & $0.8(10)$ & $0.8(10)$ & $0.9(7)$ & $0.9(7)$ \\
\hline RMS & $1.2(4)$ & $1.2(4)$ & $1.3(2)$ & $1.4(1)$ & $1.0(10)$ & $1.3(2)$ & $1.0(10)$ & $1.1(8)$ & $1.0(10)$ & $1.1(8)$ \\
\hline RMSS & $0.8(5)$ & $0.8(5)$ & $0.8(5)$ & $0.8(5)$ & $0.9(10)$ & $0.8(5)$ & $1.1(10)$ & $0.8(5)$ & $1.2(5)$ & $0.7(1)$ \\
\hline$\Sigma$ notes & 51 & 44 & 31 & 25 & 77 & 24 & 78 & 62 & 68 & 50 \\
\hline & & & & & & mber & & & & \\
\hline b & $0.6(3)$ & $0.5(1)$ & $0.6(3)$ & $0.5(1)$ & $0.7(7)$ & $0.6(3)$ & $0.8(10)$ & $0.6(3)$ & $0.7(7)$ & $0.7(7)$ \\
\hline $\mathrm{a}$ & $1.5(8)$ & $1.5(8)$ & $1.5(8)$ & $1.8(1)$ & 1.1(3) & $1.4(6)$ & $0.7(10)$ & $1.2(4)$ & $1.0(2)$ & $1.2(4)$ \\
\hline $\mathrm{R}^{2}$ & $0.8(10)$ & $0.8(10)$ & $0.8(10)$ & $0.7(1)$ & $0.8(10)$ & $0.8(10)$ & $0.7(1)$ & $0.8(10)$ & $0.8(10)$ & $0.8(10)$ \\
\hline$\overline{\mathrm{E}}$ & $0.1(1)$ & $0.1(1)$ & $0.1(1)$ & $0.1(1)$ & $0.1(1)$ & $0.1(1)$ & $0.0(10)$ & $0.1(1)$ & $0.0(10)$ & $0.1(1)$ \\
\hline SD & $1.8(8)$ & $1.9(6)$ & $1.9(6)$ & $2.1(1)$ & $1.6(10)$ & $1.8(8)$ & $2.0(4)$ & $1.6(10)$ & $1.6(10)$ & $1.6(10)$ \\
\hline $\mathrm{VC}$ & $21(3)$ & $19(5)$ & $20(4)$ & $18(6)$ & $16(7)$ & $13(10)$ & $317(1)$ & $15(9)$ & $33(2)$ & $16(8)$ \\
\hline$\overline{\mathrm{A}} \overline{\mathrm{E}}$ & $1.2(6)$ & $1.3(4)$ & $1.3(4)$ & $1.5(1)$ & $1.0(10)$ & $1.1(8)$ & $1.3(4)$ & $1.0(10)$ & $1.0(10)$ & $1.0(10)$ \\
\hline RMS & $1.8(6)$ & $1.8(6)$ & $1.8(6)$ & $2.1(1)$ & $1.6(10)$ & $1.7(8)$ & $1.9(4)$ & $1.6(10)$ & $1.6(10)$ & $1.6(10)$ \\
\hline RMSS & $0.6(5)$ & $0.5(1)$ & $0.6(5)$ & $0.5(1)$ & $0.5(1)$ & $0.5(1)$ & $1.3(10)$ & $0.5(1)$ & $0.6(5)$ & $0.5(1)$ \\
\hline$\overline{\Sigma \text { notes }}$ & 50 & 42 & 47 & 14 & 59 & 55 & 54 & 58 & 66 & 61 \\
\hline
\end{tabular}

(1) $b$, angular coefficient; a, intersection; $\mathrm{R}^{2}$, coefficient of determination; $\overline{\mathrm{E}}$, mean prediction errors; $\mathrm{SD}$, standard deviation of prediction errors; VC, coefficient of variation; $\bar{A} \bar{E}$, mean prediction absolute errors; RMS, root-mean-square prediction errors; RMSS, root-mean-square standardized prediction errors. 
Table 5. Cross-validation indicators and grades attributed (in brackets), in the second year of evaluation, obtained from ordinary kriging, for the following semivariogram models: C, circular; S, spherical; P, pentaspherical; E, exponential; G, Gaussian; R, rational quadratic; H, hole effect; K, K-Bessel; J, J-Bessel; and St, stable.

\begin{tabular}{|c|c|c|c|c|c|c|c|c|c|c|}
\hline Indicador $^{(1)}$ & $\mathrm{C}$ & $\mathrm{S}$ & $\mathrm{P}$ & $\mathrm{E}$ & $\mathrm{G}$ & $\mathrm{R}$ & $\mathrm{H}$ & $\mathrm{K}$ & $\mathrm{J}$ & St \\
\hline & \multicolumn{10}{|c|}{ October } \\
\hline $\mathrm{b}$ & $0.6(5)$ & $0.5(1)$ & $0.5(1)$ & $0.5(1)$ & $0.8(10)$ & $0.5(1)$ & $0.8(10)$ & $0.6(5)$ & $0.8(10)$ & $0.6(5)$ \\
\hline $\mathrm{a}$ & $4.3(5)$ & $4.6(4)$ & $5.3(2)$ & $5.1(3)$ & $2.6(10)$ & $5.4(1)$ & $3.3(8)$ & $4.0(7)$ & $3.1(9)$ & $4.3(5)$ \\
\hline $\mathrm{R}^{2}$ & $0.1(1)$ & $0.1(1)$ & $0.1(1)$ & $0.1(1)$ & $0.3(10)$ & $0.1(1)$ & $0.2(5)$ & $0.2(5)$ & $0.3(10)$ & $0.1(1)$ \\
\hline$\overline{\mathrm{E}}$ & $-0.6(8)$ & $-0.4(10)$ & $-0.8(5)$ & $-0.7(7)$ & $-0.9(4)$ & $-0.7(7)$ & $-1.3(2)$ & $-0.8(5)$ & $-1.7(1)$ & $-1.1(3)$ \\
\hline $\mathrm{SD}$ & $5.2(5)$ & $5.3(4)$ & $5.6(1)$ & $5.5(3)$ & $4.8(10)$ & $5.6(1)$ & $4.8(10)$ & $4.9(8)$ & $4.9(8)$ & $5.0(6)$ \\
\hline $\mathrm{VC}$ & $9(2)$ & $12(1)$ & $7(5)$ & $8(3)$ & $5(7)$ & $7(4)$ & $3(9)$ & $5(6)$ & $2(10)$ & $4(8)$ \\
\hline$\overline{\mathrm{A}} \overline{\mathrm{E}}$ & $4.3(1)$ & $4.3(1)$ & $4.3(1)$ & $4.3(1)$ & $3.7(10)$ & $4.3(1)$ & $3.8(8)$ & $4.0(6)$ & $4.0(6)$ & $4.1(4)$ \\
\hline RMS & $5.1(4)$ & $5.2(3)$ & $5.5(1)$ & $5.4(2)$ & $4.7(10)$ & $5.5(1)$ & $4.8(8)$ & $4.8(8)$ & $5.1(4)$ & $4.9(6)$ \\
\hline RMSS & $0.3(5)$ & $0.3(5)$ & $0.2(1)$ & $0.3(5)$ & $0.6(10)$ & $0.2(1)$ & $0.3(5)$ & $0.3(5)$ & $0.3(5)$ & $0.3(5)$ \\
\hline \multirow[t]{2}{*}{\begin{tabular}{|l} 
notes \\
\end{tabular}} & 37 & 30 & 18 & 26 & 81 & 18 & 65 & 55 & 63 & 43 \\
\hline & \multicolumn{10}{|c|}{ November } \\
\hline $\mathrm{b}$ & $0.5(3)$ & $0.5(3)$ & $0.4(1)$ & $0.4(1)$ & $0.7(10)$ & $0.5(3)$ & $0.7(10)$ & $0.6(7)$ & $0.7(10)$ & $0.6(7)$ \\
\hline $\mathrm{a}$ & $7.4(4)$ & $7.6(3)$ & $8.5(2)$ & $9.2(1)$ & $5.0(8)$ & $7.2(5)$ & $4.1(10)$ & $6.5(7)$ & $4.3(9)$ & $6.6(6)$ \\
\hline $\mathrm{R}^{2}$ & $0.1(5)$ & $0.1(5)$ & $0.1(5)$ & $0.0(1)$ & $0.1(5)$ & $0.1(5)$ & $0.2(10)$ & $0.1(5)$ & $0.2(10)$ & $0.1(5)$ \\
\hline$\overline{\mathrm{E}}$ & $-0.1(5)$ & $-0.2(1)$ & $-0.2(1)$ & $-0.2(1)$ & $0.0(10)$ & $-0.1(5)$ & $0.0(10)$ & $-0.1(5)$ & $0.0(10)$ & $-0.1(5)$ \\
\hline SD & $6.3(4)$ & $6.5(3)$ & $7.0(2)$ & $7.4(1)$ & $5.3(10)$ & $6.2(5)$ & $5.3(10)$ & $5.9(6)$ & $5.4(8)$ & $5.9(6)$ \\
\hline $\mathrm{VC}$ & $46(7)$ & $41(8)$ & $32(9)$ & $30(10)$ & $107(3)$ & $49(5)$ & $239(1)$ & $55(4)$ & $156(2)$ & $46(6)$ \\
\hline$\overline{\mathrm{A}} \overline{\mathrm{E}}$ & $4.6(4)$ & $4.7(3)$ & $4.9(2)$ & $5.1(1)$ & $3.8(8)$ & $4.5(5)$ & $3.7(10)$ & $4.2(7)$ & $3.8(8)$ & $4.3(6)$ \\
\hline RMS & $6.1(4)$ & $6.3(3)$ & $6.8(2)$ & $7.2(1)$ & $5.2(9)$ & $6.0(5)$ & $5.1(10)$ & $5.7(7)$ & $5.3(8)$ & $5.8(6)$ \\
\hline RMSS & $0.6(1)$ & $0.6(1)$ & $0.7(10)$ & $0.7(10)$ & $0.7(10)$ & $0.6(1)$ & $0.6(1)$ & $0.6(1)$ & $0.6(1)$ & $0.6(1)$ \\
\hline \multirow[t]{2}{*}{$\Sigma$ notes } & 37 & 30 & 34 & 27 & 73 & 39 & 72 & 49 & 66 & 48 \\
\hline & \multicolumn{10}{|c|}{ December } \\
\hline $\mathrm{b}$ & $0.6(8)$ & $0.5(6)$ & $0.4(4)$ & $0.2(1)$ & $0.7(9)$ & $0.2(1)$ & $0.7(9)$ & $0.4(4)$ & $0.8(10)$ & $0.3(2)$ \\
\hline $\mathrm{a}$ & $10.0(7)$ & $11.2(6)$ & $12.4(5)$ & $15.4(3)$ & $7.4(9)$ & $17.4(1)$ & $8.7(8)$ & $12.6(4)$ & $6.6(10)$ & $15.5(2)$ \\
\hline $\mathrm{R}^{2}$ & $0.1(10)$ & $0.0(1)$ & $0.0(1)$ & $0.0(1)$ & $0.1(10)$ & $0.0(1)$ & $0.1(10)$ & $0.0(1)$ & $0.1(10)$ & $0.0(1)$ \\
\hline$\overline{\mathrm{A}} \overline{\mathrm{E}}$ & $-1.0(5)$ & $-1.4(4)$ & $-0.8(6)$ & $-0.1(10)$ & $-1.7(3)$ & $-0.2(9)$ & $-1.8(2)$ & $-0.7(7)$ & $-3.1(1)$ & $-0.3(8)$ \\
\hline $\mathrm{SD}$ & $8.4(10)$ & $8.6(8)$ & $9.3(6)$ & $11.3(2)$ & $8.7(7)$ & $12.6(1)$ & $8.6(8)$ & $9.5(5)$ & $9.8(4)$ & $11.3(2)$ \\
\hline $\mathrm{VC}$ & $8(6)$ & $6(7)$ & $11(5)$ & $88(1)$ & $5(8)$ & $79(2)$ & $4(9)$ & $14(4)$ & $3(10)$ & $40(3)$ \\
\hline$\overline{\mathrm{A}} \overline{\mathrm{E}}$ & $6.6(10)$ & $6.9(7)$ & $7.3(4)$ & $8.7(2)$ & $6.6(10)$ & $9.6(1)$ & $6.7(8)$ & $7.3(4)$ & $7.2(6)$ & $8.7(2)$ \\
\hline RMS & $8.2(10)$ & $8.5(9)$ & $9.1(6)$ & $11.0(2)$ & $8.7(7)$ & $12.3(1)$ & $8.6(8)$ & $9.2(5)$ & $10.0(4)$ & $11.0(2)$ \\
\hline RMSS & $0.6(3)$ & $0.5(1)$ & $0.6(3)$ & $0.7(7)$ & $0.8(10)$ & $0.7(7)$ & $0.6(3)$ & $0.6(3)$ & $0.7(7)$ & $0.6(3)$ \\
\hline \multirow[t]{2}{*}{$\Sigma$ notes } & 69 & 49 & 40 & 28 & 73 & 24 & 65 & 33 & 62 & 25 \\
\hline & \multicolumn{10}{|c|}{ January } \\
\hline $\mathrm{b}$ & $0.5(7)$ & $0.5(7)$ & $0.5(7)$ & $0.4(3)$ & $0.4(3)$ & $0.2(1)$ & $0.6(10)$ & $0.4(3)$ & $0.6(10)$ & $0.4(3)$ \\
\hline $\mathrm{a}$ & $9.9(8)$ & $10.2(7)$ & $10.2(7)$ & $12.1(2)$ & $10.6(4)$ & $15.2(1)$ & $7.5(10)$ & $10.5(5)$ & $8.1(9)$ & $10.7(3)$ \\
\hline $\mathrm{R}^{2}$ & $0.0(1)$ & $0.0(1)$ & $0.0(1)$ & $0.0(1)$ & $0.0(1)$ & $0.0(1)$ & $0.1(10)$ & $0.0(1)$ & $0.1(10)$ & $0.0(1)$ \\
\hline$\overline{\mathrm{E}}$ & $0.6(3)$ & $0.4(8)$ & $0.5(7)$ & $0.1(10)$ & $0.3(9)$ & $0.6(3)$ & $0.7(1)$ & $-0.4(8)$ & $0.7(1)$ & $0.5(7)$ \\
\hline SD & $8.2(5)$ & $8.3(3)$ & $8.3(3)$ & $8.6(2)$ & $7.7(8)$ & $9.7(1)$ & $6.8(10)$ & $7.8(7)$ & $7.3(9)$ & $8.2(5)$ \\
\hline $\mathrm{VC}$ & $13(8)$ & $20(4)$ & $15(7)$ & $76(1)$ & $22(2)$ & $15(6)$ & $9(10)$ & $20(3)$ & $10(9)$ & $16(5)$ \\
\hline$\overline{\mathrm{A}} \overline{\mathrm{E}}$ & $6.0(5)$ & $6.1(3)$ & $6.1(3)$ & $6.5(2)$ & $5.8(8)$ & $7.4(1)$ & $4.8(10)$ & $5.9(7)$ & $5.2(9)$ & $6.0(5)$ \\
\hline RMS & $8.0(5)$ & $8.1(3)$ & $8.1(3)$ & $8.4(2)$ & $7.5(8)$ & $9.5(1)$ & $6.7(10)$ & $7.6(7)$ & $7.1(9)$ & $8.0(5)$ \\
\hline RMSS & $1.3(1)$ & $1.2(3)$ & $1.3(1)$ & $0.9(7)$ & $0.9(7)$ & $1.0(10)$ & $1.3(1)$ & $0.9(7)$ & $1.3(1)$ & $1.1(7)$ \\
\hline \multirow[t]{2}{*}{$\sum$ notes } & 43 & 39 & 39 & 30 & 40 & 25 & 72 & 47 & 67 & 41 \\
\hline & \multicolumn{10}{|c|}{ February } \\
\hline $\mathrm{b}$ & $0.6(7)$ & $0.6(7)$ & $0.6(7)$ & $0.5(3)$ & $0.7(10)$ & $0.4(1)$ & $0.7(10)$ & $0.6(7)$ & $0.7(10)$ & $0.6(7)$ \\
\hline $\mathrm{a}$ & $10.7(5)$ & $10.9(4)$ & $10.7(5)$ & $12.9(2)$ & $9.3(8)$ & $15.0(1)$ & $7.2(10)$ & $9.6(7)$ & $8.6(9)$ & $9.6(7)$ \\
\hline $\mathrm{R}^{2}$ & $0.1(10)$ & $0.1(10)$ & $0.1(10)$ & $0.0(1)$ & $0.1(10)$ & $0.0(1)$ & $0.1(10)$ & $0.1(10)$ & $0.1(10)$ & $0.1(10)$ \\
\hline$\overline{\mathrm{E}}$ & $0.3(4)$ & $0.2(6)$ & $-0.1(8)$ & $-0.1(8)$ & $0.0(10)$ & $0.3(4)$ & $-0.2(6)$ & $-0.4(2)$ & $-0.5(1)$ & $0.4(2)$ \\
\hline SD & $9.7(5)$ & $9.8(4)$ & $10.2(3)$ & $10.2(3)$ & $9.0(10)$ & 11.3(1) & $9.3(7)$ & $9.3(7)$ & $9.5(6)$ & $9.2(8)$ \\
\hline $\mathrm{VC}$ & $30(4)$ & $39(5)$ & $112(8)$ & $126(9)$ & $433(10)$ & $42(6)$ & $59(7)$ & $25(3)$ & $18(1)$ & $23(2)$ \\
\hline$\overline{\mathrm{A}} \overline{\mathrm{E}}$ & $7.3(5)$ & $7.3(5)$ & $7.6(3)$ & $7.7(2)$ & $6.5(10)$ & $8.3(1)$ & $7.0(7)$ & $7.0(7)$ & $7.3(5)$ & $6.8(9)$ \\
\hline RMS & $9.4(5)$ & $9.6(4)$ & $9.9(3)$ & $10.0(2)$ & $8.7(10)$ & $11.0(1)$ & $9.0(8)$ & $9.1(7)$ & $9.2(6)$ & $8.9(9)$ \\
\hline RMSS & $1.1(7)$ & $1.1(7)$ & $1.0(10)$ & $0.9(7)$ & $1.1(7)$ & $1.0(10)$ & $1.5(1)$ & $1.1(7)$ & $1.4(3)$ & $1.0(10)$ \\
\hline \multirow[t]{2}{*}{$\Sigma$ notes } & 52 & 52 & 57 & 37 & 85 & 26 & 66 & 57 & 51 & 64 \\
\hline & & & & & & & & & & \\
\hline $\mathrm{b}$ & $0.7(10)$ & $0.5(3)$ & $0.4(1)$ & $0.6(7)$ & $0.6(7)$ & $0.6(7)$ & $0.7(10)$ & $0.6(7)$ & $0.7(10)$ & $0.6(7)$ \\
\hline $\mathrm{a}$ & $20.4(8)$ & $35.6(4)$ & $49.2(1)$ & $34.7(5)$ & $20.5(7)$ & $26.3(6)$ & $13.1(10)$ & $36.0(3)$ & $15.0(9)$ & $36.3(2)$ \\
\hline $\mathrm{R}^{2}$ & $0.0(1)$ & $0.0(1)$ & $0.0(1)$ & $0.0(1)$ & $0.0(1)$ & $0.0(1)$ & $0.1(10)$ & $0.0(1)$ & $0.0(1)$ & $0.0(1)$ \\
\hline$\overline{\mathrm{E}}$ & $-13.3(1)$ & $6.1(7)$ & $5.2(9)$ & $-6.1(7)$ & $-9.5(3)$ & $-3.7(10)$ & $-9.1(4)$ & $8.5(6)$ & $-8.9(5)$ & $-9.9(2)$ \\
\hline SD & $63.3(5)$ & $73.6(2)$ & $82.8(1)$ & $67.1(4)$ & $48.0(8)$ & $62.4(6)$ & $37.5(10)$ & $68.0(3)$ & $40.9(9)$ & $53.4(7)$ \\
\hline $\mathrm{VC}$ & $4(9)$ & $12(3)$ & $16(2)$ & $10(4)$ & $5(7)$ & $17(1)$ & $3(10)$ & $8(5)$ & $4(8)$ & $5(6)$ \\
\hline$\overline{\mathrm{A}} \overline{\mathrm{E}}$ & $43.9(2)$ & $38.3(5)$ & $52.3(1)$ & $42.1(3)$ & $29.2(8)$ & $38.3(5)$ & $24.2(10)$ & $35.8(6)$ & $25.5(9)$ & $31.8(7)$ \\
\hline RMS & $63.0(5)$ & $71.9(2)$ & $80.7(1)$ & $65.6(4)$ & $47.7(8)$ & $60.8(6)$ & $37.6(10)$ & $66.8(3)$ & $40.8(9)$ & $53.0(7)$ \\
\hline RMSS & $0.3(2)$ & $0.6(5)$ & $0.5(4)$ & $0.4(3)$ & $0.8(8)$ & $0.4(3)$ & $2.0(1)$ & $0.9(10)$ & $1.2(8)$ & $0.7(6)$ \\
\hline$\overline{\Sigma \text { notes }}$ & 43 & 32 & 19 & 38 & 57 & 45 & 75 & 44 & 68 & 45 \\
\hline
\end{tabular}

Continues... 
Table 5. Continuation...

\begin{tabular}{|c|c|c|c|c|c|c|c|c|c|c|}
\hline \multirow[b]{2}{*}{$\mathrm{b}$} & \multicolumn{10}{|c|}{ April } \\
\hline & $0.9(10)$ & $0.9(10)$ & $0.9(10)$ & $0.9(10)$ & $0.9(10)$ & $0.9(10)$ & $0.9(10)$ & $0.9(10)$ & $0.9(10)$ & $0.9(10)$ \\
\hline $\mathrm{a}$ & $2.7(5)$ & $3.6(4)$ & $3.9(3)$ & 5.1(1) & $1.3(8)$ & $4.9(2)$ & $2.7(5)$ & $1.2(10)$ & $1.4(7)$ & $1.2(10)$ \\
\hline $\mathrm{R}^{2}$ & $0.3(4)$ & $0.3(4)$ & $0.2(7)$ & $0.2(7)$ & $0.7(1)$ & $0.2(7)$ & $0.3(4)$ & $0.8(10)$ & $0.6(2)$ & $0.8(10)$ \\
\hline$\overline{\mathrm{E}}$ & $-1.5(7)$ & $-2.6(5)$ & $-3.0(2)$ & $-3.6(1)$ & $1.1(10)$ & $-2.7(4)$ & $-2.8(3)$ & $1.1(10)$ & $-2.4(6)$ & $1.1(10)$ \\
\hline SD & $10.9(7)$ & $11.1(5)$ & $11.6(4)$ & $12.4(3)$ & $10.3(10)$ & $11.1(5)$ & $17.9(1)$ & $10.3(10)$ & $13.8(2)$ & $10.3(10)$ \\
\hline $\mathrm{VC}$ & $7(4)$ & $4(7)$ & $3(9)$ & $3(10)$ & $9(2)$ & $4(8)$ & $6(5)$ & $9(1)$ & $5(6)$ & $9(2)$ \\
\hline$\overline{\mathrm{A}} \overline{\mathrm{E}}$ & $9.5(6)$ & $9.8(5)$ & $9.9(4)$ & $10.7(2)$ & $7.9(10)$ & $9.2(7)$ & 13.1(1) & $7.9(10)$ & $10.3(3)$ & $7.9(10)$ \\
\hline RMS & $10.7(7)$ & $11.1(5)$ & $11.6(4)$ & $12.6(3)$ & $10.1(10)$ & $11.1(5)$ & $17.6(1)$ & $10.1(10)$ & $13.7(2)$ & $10.1(10)$ \\
\hline RMSS & $0.5(7)$ & $0.4(3)$ & $0.4(3)$ & $0.4(3)$ & $1.4(8)$ & $0.4(3)$ & $2.0(1)$ & $1.3(10)$ & $2.0(1)$ & $1.4(8)$ \\
\hline \multirow[t]{2}{*}{$\sum$ notes } & 57 & 48 & 46 & 40 & 69 & 51 & 31 & 81 & 46 & 80 \\
\hline & \multicolumn{10}{|c|}{ May } \\
\hline b & $0.5(5)$ & $0.5(5)$ & $0.3(3)$ & $0.3(3)$ & $0.7(8)$ & $0.2(1)$ & $0.8(10)$ & $0.7(8)$ & $0.8(10)$ & $0.7(8)$ \\
\hline $\mathrm{a}$ & $11.0(4)$ & $11.0(4)$ & $15.3(3)$ & $17.2(2)$ & $5.2(8)$ & $20.7(1)$ & $3.9(10)$ & $7.1(6)$ & $5.0(9)$ & $6.7(7)$ \\
\hline $\mathrm{R}^{2}$ & $0.0(1)$ & $0.0(1)$ & $0.0(1)$ & $0.0(1)$ & $0.1(5)$ & $0.0(1)$ & $0.2(10)$ & $0.1(5)$ & $0.2(10)$ & $0.1(5)$ \\
\hline$\overline{\mathrm{A}} \overline{\mathrm{E}}$ & $0.9(6)$ & $1.0(5)$ & $0.6(7)$ & $0.5(8)$ & $-1.7(3)$ & $0.4(10)$ & $-0.4(10)$ & $-1.9(1)$ & $-1.5(4)$ & $-1.9(1)$ \\
\hline SD & $11.3(8)$ & $11.3(8)$ & $12.0(3)$ & $12.1(2)$ & $10.9(9)$ & $13.2(1)$ & $10.2(10)$ & $11.5(6)$ & $11.2(9)$ & $11.6(5)$ \\
\hline $\mathrm{VC}$ & $12(5)$ & $11(6)$ & $18(4)$ & $25(3)$ & $6(8)$ & $33(1)$ & $25(2)$ & $6(9)$ & $7(7)$ & $6(10)$ \\
\hline$\overline{\mathrm{A}} \overline{\mathrm{E}}$ & $8.4(6)$ & $8.3(7)$ & $9.8(3)$ & $10.0(2)$ & $8.1(9)$ & 11.1(1) & $7.3(10)$ & $8.2(8)$ & $8.6(5)$ & $8.3(7)$ \\
\hline RMS & 11.1(6) & $11.0(7)$ & $11.7(3)$ & $11.8(2)$ & $10.8(9)$ & $12.8(1)$ & $9.9(10)$ & $11.3(5)$ & $11.0(7)$ & $11.4(4)$ \\
\hline RMSS & $1.1(10)$ & $1.1(10)$ & $1.2(7)$ & $1.1(10)$ & $2.0(1)$ & $1.1(10)$ & $2.0(1)$ & $1.7(5)$ & $2.0(1)$ & $1.8(3)$ \\
\hline \multirow[t]{2}{*}{$\sum$ notes } & 51 & 53 & 34 & 33 & 60 & 27 & 73 & 53 & 62 & 50 \\
\hline & \multicolumn{10}{|c|}{ June } \\
\hline b & $0.3(10)$ & $0.3(10)$ & $0.3(10)$ & $0.2(1)$ & $0.3(10)$ & $0.2(1)$ & $0.3(10)$ & $0.2(1)$ & $0.3(10)$ & $0.3(10)$ \\
\hline $\mathrm{a}$ & $8.5(8)$ & $9.2(6)$ & $9.6(2)$ & $11.0(1)$ & $9.2(6)$ & $9.3(4)$ & $7.6(10)$ & $9.4(3)$ & $8.1(9)$ & $9.1(7)$ \\
\hline $\mathrm{R}^{2}$ & $0.0(10)$ & $0.0(10)$ & $0.0(10)$ & $0.0(10)$ & $0.0(10)$ & $0.0(10)$ & $0.0(10)$ & $0.0(10)$ & $0.0(10)$ & $0.0(10)$ \\
\hline$\overline{\mathrm{E}}$ & $2.2(9)$ & $2.4(7)$ & $2.2(9)$ & $2.0(10)$ & $-2.4(7)$ & $2.6(3)$ & $-2.7(2)$ & $2.5(5)$ & $-2.8(1)$ & $2.5(5)$ \\
\hline $\mathrm{SD}$ & $9.6(8)$ & $10.0(5)$ & $10.0(5)$ & $10.7(1)$ & $9.9(7)$ & $10.2(2)$ & $9.5(10)$ & $10.1(4)$ & $9.5(10)$ & $10.0(5)$ \\
\hline $\mathrm{VC}$ & $47(3)$ & $46(5)$ & $42(2)$ & $52(1)$ & $46(5)$ & $39(8)$ & $36(9)$ & $44(6)$ & $34(10)$ & $40(7)$ \\
\hline$\overline{\mathrm{A}} \overline{\mathrm{E}}$ & $7.2(8)$ & $7.5(5)$ & $7.5(5)$ & $8.0(1)$ & $7.4(7)$ & $7.7(2)$ & $7.1(9)$ & $7.6(3)$ & $7.0(10)$ & $7.5(5)$ \\
\hline RMS & $9.6(10)$ & $10.0(6)$ & $10.0(6)$ & $10.6(1)$ & $10.0(6)$ & $10.2(2)$ & $9.7(8)$ & $10.1(4)$ & $9.7(8)$ & $10.0(6)$ \\
\hline RMSS & $1.1(5)$ & $1.0(10)$ & $0.9(5)$ & $0.9(5)$ & $1.0(10)$ & $1.0(10)$ & $1.3(1)$ & $1.0(10)$ & $1.0(10)$ & $1.0(10)$ \\
\hline \multirow[t]{2}{*}{$\sum$ notes } & 71 & 64 & 54 & 31 & 63 & 42 & 69 & 46 & 78 & 65 \\
\hline & \multicolumn{10}{|c|}{ July } \\
\hline $\mathrm{b}$ & $0.5(6)$ & $0.4(4)$ & $0.4(4)$ & $0.2(1)$ & $0.6(8)$ & $0.2(1)$ & $0.7(10)$ & $0.3(2)$ & $0.4(4)$ & $0.3(2)$ \\
\hline $\mathrm{a}$ & $3.2(8)$ & $3.6(6)$ & $3.9(5)$ & $5.1(1)$ & $2.0(10)$ & $4.9(2)$ & $2.7(9)$ & $4.3(3)$ & $3.5(7)$ & $4.3(3)$ \\
\hline $\mathrm{R}^{2}$ & $0.1(5)$ & $0.1(5)$ & $0.1(5)$ & $0.0(1)$ & $0.3(10)$ & $0.0(1)$ & $0.3(10)$ & $0.1(5)$ & $0.1(5)$ & $0.1(5)$ \\
\hline$\overline{\mathrm{E}}$ & $0.7(5)$ & $0.7(5)$ & $0.6(8)$ & $0.5(9)$ & $0.7(5)$ & $0.7(5)$ & $-0.2(10)$ & $0.7(5)$ & $0.9(1)$ & $0.7(5)$ \\
\hline SD & $4.1(10)$ & $4.2(8)$ & $4.3(6)$ & $4.9(3)$ & $4.2(8)$ & $4.9(3)$ & $5.4(1)$ & $4.5(5)$ & $4.3(6)$ & $4.6(4)$ \\
\hline $\mathrm{VC}$ & $6(7)$ & $6(7)$ & $7(5)$ & $9(2)$ & $5(9)$ & $8(3)$ & $21(1)$ & $7(5)$ & $4(10)$ & $7(5)$ \\
\hline$\overline{\mathrm{A}} \overline{\mathrm{E}}$ & $3.0(8)$ & $3.1(7)$ & $3.2(5)$ & $3.7(1)$ & $2.9(9)$ & $3.5(2)$ & $3.2(5)$ & $3.3(3)$ & $2.7(10)$ & $3.3(3)$ \\
\hline RMS & $4.0(10)$ & $4.1(9)$ & $4.2(8)$ & $4.8(3)$ & $4.2(8)$ & $4.8(3)$ & $5.2(1)$ & $4.5(5)$ & $4.3(6)$ & $4.5(5)$ \\
\hline RMSS & $0.8(7)$ & $0.7(3)$ & $0.6(1)$ & $0.7(3)$ & $0.9(10)$ & $0.7(3)$ & 1.1(10) & $0.7(3)$ & $0.7(3)$ & $0.7(3)$ \\
\hline \multirow[t]{2}{*}{$\sum$ notes } & 66 & 54 & 47 & 24 & 77 & 23 & 57 & 36 & 52 & 34 \\
\hline & \multicolumn{10}{|c|}{ August } \\
\hline b & $0.3(3)$ & $0.3(3)$ & $0.2(1)$ & $0.2(1)$ & $0.5(10)$ & $0.2(1)$ & $0.5(10)$ & $0.3(3)$ & $0.4(7)$ & $0.3(3)$ \\
\hline $\mathrm{a}$ & $3.0(6)$ & $3.1(4)$ & $3.3(3)$ & $3.8(1)$ & $1.8(10)$ & $3.4(2)$ & $1.8(10)$ & $3.0(6)$ & $2.2(8)$ & $2.9(7)$ \\
\hline $\mathrm{R}^{2}$ & $0.1(3)$ & $0.1(3)$ & $0.1(3)$ & $0.0(1)$ & $0.3(10)$ & $0.1(3)$ & $0.3(10)$ & $0.1(3)$ & $0.2(7)$ & $0.1(3)$ \\
\hline$\overline{\mathrm{E}}$ & $0.7(3)$ & $0.6(7)$ & $0.6(7)$ & $0.4(10)$ & $0.9(1)$ & $0.6(7)$ & $0.7(3)$ & $0.7(3)$ & $0.9(1)$ & $0.7(3)$ \\
\hline SD & $2.8(6)$ & $2.9(4)$ & $2.9(4)$ & $3.1(1)$ & $2.6(10)$ & $3.0(2)$ & $2.8(6)$ & $2.8(6)$ & $2.6(10)$ & $2.7(8)$ \\
\hline $\mathrm{VC}$ & $41(5)$ & $46(4)$ & $52(2)$ & $75(1)$ & $30(10)$ & $51(3)$ & $39(8)$ & $40(7)$ & $31(9)$ & $40(7)$ \\
\hline$\overline{\mathrm{A}} \overline{\mathrm{E}}$ & $2.3(5)$ & $2.4(3)$ & $2.4(3)$ & $2.6(1)$ & $2.0(10)$ & $2.4(3)$ & $2.2(7)$ & $2.3(5)$ & $2.1(9)$ & $2.2(7)$ \\
\hline RMS & $2.8(6)$ & $2.8(6)$ & $2.9(3)$ & $3.1(1)$ & $2.6(10)$ & $2.9(3)$ & $2.9(3)$ & $2.8(6)$ & $2.7(8)$ & $2.7(8)$ \\
\hline RMSS & $0.9(7)$ & $0.9(7)$ & $0.8(5)$ & $0.9(7)$ & $1.4(1)$ & $0.9(7)$ & $1.3(3)$ & $0.9(7)$ & $1.0(10)$ & $0.9(7)$ \\
\hline \multirow[t]{2}{*}{$\sum$ notes } & 44 & 41 & 31 & 24 & 72 & 31 & 60 & 46 & 69 & 53 \\
\hline & & & & & & & & & & \\
\hline $\mathrm{b}$ & $0.2(3)$ & $0.2(3)$ & $0.2(3)$ & $0.1(1)$ & $0.3(7)$ & $0.1(1)$ & $0.4(10)$ & $0.1(1)$ & $0.4(10)$ & $0.3(7)$ \\
\hline $\mathrm{a}$ & $2.9(6)$ & $3.2(5)$ & $3.3(4)$ & $3.6(2)$ & $2.1(7)$ & $4.5(1)$ & $1.6(10)$ & $3.5(3)$ & $1.7(9)$ & $2.1(7)$ \\
\hline $\mathrm{R}^{2}$ & $0.1(3)$ & $0.1(3)$ & $0.0(1)$ & $0.0(1)$ & $0.2(7)$ & $0.0(1)$ & $0.3(10)$ & $0.0(1)$ & $0.2(7)$ & $0.2(7)$ \\
\hline$\overline{\mathrm{E}}$ & $0.9(4)$ & $0.8(6)$ & $0.7(8)$ & $0.6(10)$ & $-1.2(1)$ & $0.7(8)$ & $1.1(2)$ & $0.7(8)$ & $1.1(2)$ & $1.2(1)$ \\
\hline SD & $3.7(5)$ & $3.8(4)$ & $3.9(3)$ & $4.0(1)$ & $3.2(7)$ & $4.0(1)$ & $2.8(10)$ & $3.9(3)$ & $2.9(9)$ & $3.2(7)$ \\
\hline $\mathrm{VC}$ & $40(6)$ & $46(5)$ & $53(4)$ & $65(1)$ & $26(7)$ & $54(3)$ & $25(10)$ & $57(2)$ & $26(7)$ & $26(7)$ \\
\hline$\overline{\mathrm{A}} \overline{\mathrm{E}}$ & $2.8(5)$ & $2.9(3)$ & $2.9(3)$ & $3.0(1)$ & $2.5(7)$ & $2.9(3)$ & $2.2(10)$ & $2.9(3)$ & $2.3(9)$ & $2.5(7)$ \\
\hline RMS & $3.7(7)$ & $3.8(5)$ & $3.8(5)$ & $4.0(1)$ & $3.3(8)$ & $3.9(3)$ & $3.0(10)$ & $3.9(3)$ & $3.1(9)$ & $3.3(8)$ \\
\hline RMSS & $0.8(3)$ & $0.7(1)$ & $0.7(1)$ & $0.7(1)$ & $1.0(10)$ & $0.7(1)$ & $1.0(10)$ & $0.7(1)$ & $0.9(7)$ & $1.0(10)$ \\
\hline$\Sigma$ notes & 42 & 35 & 32 & 19 & 61 & 22 & 82 & 25 & 69 & 61 \\
\hline
\end{tabular}

${ }^{(1)} \mathrm{b}$, angular coefficient; a, intersection; $\mathrm{R}^{2}$, coefficient of determination; $\overline{\mathrm{E}}$, mean prediction errors; SD, standard deviation of prediction errors; VC, coefficient of variation; $\overline{\mathrm{A}} \overline{\mathrm{E}}$, mean prediction absolute errors; RMS, root-mean-square prediction errors; RMSS, root-mean-square standardized prediction errors. 
The variability of models obtained for each month reflects the influence of the evaluation time on the spatial distribution of fig fly population density. Between the first and second years, only three months show the same selection of semivariogram models (January, March, and April). However, it would take more years to make a proper comparison among the selected models throughout the months.

The selected models did not present nugget effect, indicating the absence of discontinuity in the semivariogram models, for distances smaller than the smallest distance among the samples $(13 \mathrm{~m}$ in the present case, between the points 6 and 7) (Webster \& Oliver, 2007).

The range of values obtained with the selected semivariogram models were quite variable over the years and months $(\geq 42.2 \mathrm{~m})$. In the first year, the lowest values were obtained in October, November, and July, and the highest, in December and February. In the second year, the lowest values were observed in October, November, and December, and the highest, in January. In average, range values were superior in the second year (Tables 2 and 3). However, the reason for this variation could not be explained, since the values of descriptive statistics bear no relation to the estimated parameters of the theoretical semivariogram models. Nielsen \& Wendroth (2002) found that the variation range represents the maximum distance of spatial autocorrelation, indicating that the points located in an area whose radius is in its range are more similar to each other than those separated by greater distances. According to Webster \& Oliver (2007), the range constitutes the maximum distance of spatial dependence. The obtained results indicate that the distances among the traps were adequate, and that it is possible to determine a rearrangement of trapping range from the results of the smallest range of the selected models.

\section{Conclusions}

1. Each data set (months) of fig fly population density has a particular spatial dependence structure, which makes it necessary to define specific models of theoretical semivariograms in order to enhance the adjustment to the experimental semivariogram.

2. It was not possible to determine a standard theoretical semivariogram model; instead, six theoretical models were selected: circular, Gaussian, hole effect, K-Bessel, J-Bessel, and stable.

\section{Acknowledgements}

To Conselho Nacional de Desenvolvimento Científico e Tecnológico (CNPq) and to Coordenação de Aperfeiçoamento de Pessoal de Nível Superior (Capes), for scholarships granted; and to Dionísio Link (in memoriam), for research orientation.

\section{References}

BOTTEGA, E.L.; QUEIROZ, D.M. de; PINTO, F. de A. de C.; SOUZA, C.M.A. de. Variabilidade espacial de atributos do solo em sistema de semeadura direta com rotação de culturas no cerrado brasileiro. Revista Ciência Agronômica, v.44, p.1-9, 2013. DOI: 10.1590/S1806-66902013000100001.

BOX, G.E.P.; COX, D.R. Analysis of transformations. Journal of the Royal Statistical Society, v.26, p.211-243, 1964.

DINARDO-MIRANDA, L.L.; FRACASSO, J.V. Spatial and temporal variability of plant-parasitic nematodes population in sugarcane. Bragantia, v.69, p.39-52, 2010. DOI: 10.1590/ S0006-87052010000500006.

ELLSBURY, M.M.; WOODSON, W.D.; CLAY, S.A.; MALO, D.; SCHUMACHER, J.; CLAY, D.E.; CARLSON, C.G. Geostatistical characterization of the spatial distribution of adult corn rootworm (Coleoptera: Chrysomelidae) emergence. Environmental Entomology, v.27, p.910-917, 1998.

FARACO, M.A.; URIBE-OPAZO, M.A.; SILVA, E.A.A da; JOHANN, J.A.; BORSSOI, J.A. Seleção de modelos de variabilidade espacial para elaboração de mapas temáticos de atributos físicos do solo e produtividade da soja. Revista Brasileira de Ciência do Solo, v.32, p.463-476, 2008. DOI: 10.1590/S0100-06832008000200001

FARIAS, P.R.S.; BARBOSA, J.C.; BUSOLI, A.C.; OVERAL, W.L.; MIRANDA, V.S.; RIBEIRO, S.M. Spatial analysis of the distribution of Spodoptera frugiperda (J.E. Smith) (Lepidoptera: Noctuidae) and losses in maize crop productivity using geostatistics. Neotropical Entomology, v.37, p.321-327, 2008. DOI: 10.1590/ S1519-566X2008000300012.

GOOVAERTS, P. Geostatistics for natural resources evaluation. New York: Oxford University Press, 1997. 496p.

GUNDOGDU, K.S.; GUNEY, I. Spatial analyses of groundwater levels using universal kriging. Journal of Earth System Science, v.116, p.49-55, 2007. DOI: 10.1007/s12040-007-0006-6.

HELDWEIN, A.B.; BURIOL, A.G.; STRECK, N.A. O clima de Santa Maria. Ciência e Ambiente, v.38, p.43-58, 2009.

JOHNSON, K; VER HOEF, J.M.; KRIVORUCHKO, K.; LUCAS, N. ArcGIS 9: using ArcGIS geostatistical analyst. Redlands: ESRI, 2001. 306p.

LASMAR, O.; ZANETTI, R.; DOS SANTOS, A.; FERNANDES, B.V. Use of geostatistics to determine the spatial distribution 
and infestation rate of leaf-cutting ant nests (Hymenoptera: Formicidae) in eucalyptus plantations. Neotropical Entomology, v.41, p.324-332, 2012. DOI: 10.1007/s13744-012-0040-1.

MELLO, J.M. de; BATISTA, J.L.F.; RIBEIRO JÚNIOR, P.J.; OLIVEIRA, M.S. de. Ajuste e seleção de modelos espaciais de semivariograma visando à estimativa volumétrica de Eucalyptus grandis. Scientia Forestalis, v.69, p.25-37, 2005.

MORA, A.; BEER, J. Geostatistical modeling of the spatial variability of coffee fine roots under Erythrina shade trees and contrasting soil management. Agroforestry Systems, v.87, p.365-376, 2013. DOI: 10.1007/s10457-012-9557-x.

NIELSEN, D.R.; WENDROTH, O. Spatial and temporal statistics. Reiskirchen: Catena Verlag GMBH, 2002. 398p.

NOETZOLD, R.; ALVES, M. de C.; CASSETARI NETO, D.; MACHADO, A.Q. Variabilidade espacial de Colletotrichum truncatum em campo de soja sob três níveis de sanidade de sementes. Summa Phytopathologica, v.40, p.16-23, 2014. DOI: 10.1590/S0100-54052014000100002.

PASINI, M.P.B.; LINK, D. Efficiency of different traps to capture Zaprionus indianus (Diptera: Drosophilidae) in fig orchard in Santa Maria county, Rio Grande do Sul State, Brazil. International Research Journal of Agricultural Science and Soil Science, v.1, p.349-354, 2011.
PASINI, M.P.B.; LINK, D.; SCHAICH, G. Attractive solutions efficiency in capturing Zaprionus indianus Gupta, 1970 (Diptera: Drosophilidae) in Ficus carica L. (Moraceae) orchard in Santa Maria, Rio Grande do Sul, Brazil. Entomotropica, v.26, p.107-116, 2011.

RAGA, A.; MACHADO, R.A.; DINARDO, W.; STRIKIS, P.C. Eficácia de atrativos alimentares na captura de moscas-das-frutas em pomar de citros. Bragantia, v.65, p.337-345, 2006. DOI: 10.1590/S0006-87052006000200016.

SILVA, A.F. da; LIMA, J.S. de S.; OLIVEIRA, R.B. de. Métodos de interpolação para estimar o $\mathrm{pH}$ em solo sob dois manejos de café arábica. Idesia, v.28, p.61-66, 2010. DOI: 10.4067/ S0718-34292010000200007.

WEBSTER, R.; OLIVER, M.A. Geostatistics for environmental scientists. $2^{\text {nd }}$ ed. Chichester: J. Wiley, 2007. 330p. DOI: 10.1002/9780470517277.

YAMAMOTO, J.K.; LANDIM, P.M.B. Geoestatística: conceitos e aplicações. São Paulo: Oficina de textos, 2013. 215p.

YASSIN, A.; DAVID, J.R. Revision of the afrotropical species of Zaprionus (Diptera, Drosophilidae), with descriptions of two new species and notes on internal reproductive structures and immature stages. Zookeys, v.51, p.33-72, 2010. DOI: 10.3897/ zookeys.51.380.

$\overline{\text { Received on January 10, } 2014 \text { and accepted on June 16, } 2014}$ 